\title{
22. HIGH-RESOLUTION STRATIGRAPHIC CORRELATION OF ODP LEG 111 HOLES 677A AND 677B AND DSDP LEG 69 HOLE 504 1
}

\author{
Joanne M. Alexandrovich and James D. Hays²
}

\begin{abstract}
The recovery of continuous sedimentary sequences has been and is a high-priority goal of the Ocean Drilling Program, yet its success has not been determined. We test the stratigraphic continuity of continuously cored sequences from ODP Leg 111 Holes 677A and 677B and DSDP Leg 69 Hole 504. The two holes at Site 677 and Hole 504 are correlated using high-resolution biostratigraphy, tephra stratigraphy, and time series of opaline silica and calcium carbonate. The correlation coefficient between the time series is maximized through an inverse correlation procedure. Changes in slope of the mapping function that relates the two sections indicate gaps or changes in the accumulation rate between sections. We assume as a first approximation that the sedimentary sequences are identical in each hole, given their close proximity. Slope changes of the mapping functions therefore result from errors introduced by coring. This assumption is justified by the fact that most mapping function slope changes occur at core breaks. Having identified missing sections in one site, it is possible to fill these gaps with the appropriate section from the adjacent site, thereby improving continuity. This is possible only where the sediment gaps identified at core breaks in one hole do not correspond to core break levels in the other hole. Results show that in the upper $100 \mathrm{~m}$ of the stratigraphic column, $3.1 \%$ and $4.6 \%$ were unsampled in Holes $677 \mathrm{~A}$ and $677 \mathrm{~B}$, respectively, and $15 \%$ of the Pliocene portion of Hole 504 was not sampled by the hydraulic piston corer. This indicates that even in relatively calm seas, double or triple coring is required in order to ensure that gaps and doubly sampled intervals of the stratigraphic column which occur at core breaks are recognized and sampled at least once.
\end{abstract}

\section{INTRODUCTION}

The goal of sediment coring on Leg 111 was to retrieve a complete stratigraphic section for high-resolution biostratigraphic and paleoceanographic studies (Shipboard Scientific Party, 1988). Complete stratigraphic sections and accurate age models are necessary foundations for many geologic investigations, such as the study of evolutionary patterns and rates and determining the timing of continental ice buildup and sea-level change. Once an accurate composite stratigraphy is established for Ocean Drilling Program (ODP) Site 677, paleoceanographic scenarios and paleoclimatic events can be placed in a chronological framework and used to examine and interpret the interrelationships between this site and other deep-sea and continental records.

The efforts of the Deep Sea Drilling Project (DSDP) and ODP have expanded the opportunity for paleoceanographic studies on many time scales by supplying records of marine sedimentation spanning the last 200 m.y. Many sites have been continuously and double cored; however, few attempts have been made to quantify exactly how well the techniques used, specifically the hydraulic piston corer, actually sample a stratigraphic column. Ruddiman et al. (1986) used core color to identify correlative horizons and have subsequently (Ruddiman et al., 1989; Raymo et al., 1989) used this technique, enhanced with calcium carbonate data, to splice double-cored holes together to obtain a more complete composite section. Their composite stratigraphy is based solely on visual correlation of color and calcium carbonate variations, and they do not discuss how much of the stratigraphic column was not sampled or sampled twice. Here, using the sediments cored during ODP Leg 111 which cover late Miocene to Holocene time, we will determine the completeness of sedimentary sections from DSDP Hole 504 and ODP Holes

\footnotetext{
${ }^{1}$ Becker, K., Sakai, H., et al., 1989. Proc. ODP, Sci. Results, 111: College Station, TX (Ocean Drilling Program).

2 Department of Geological Sciences, Columbia University, and LamontDoherty Geological Observatory of Columbia University, Palisades, NY 10964.
}

677A and 677B using several strategies and establish a composite section that can provide a solid stratigraphic basis for detailed paleoceanographic studies.

The development of the hydraulic piston corer enables the retrieval of relatively undisturbed sediments with reported recovery rates that are quite high. With recovery of over $100 \%$, as recorded for the Pleistocene and upper Pliocene sediments of Site 677 , it might be questioned whether double piston coring is really necessary. Site 677 was double cored, and in combination with nearby Site 504, these sections provide a fine opportunity to test the usefulness of double and/or triple coring in a region where high-resolution correlations have been shown to be possible (Dunn and Moore, 1981). We also examine how gaps in stratigraphic records can be objectively recognized and quantified using biostratigraphic, lithologic, and geochemical data.

The approach we take is as follows. First, biostratigraphic datum levels and tephra layers are used as primary stratigraphic control. The datum levels are assumed to be synchronous between holes within the sampling interval. Second, downcore percent calcium carbonate and percent $\mathrm{Si}$-opal records are used as input signals to CORPAC (CORrelation PACkage), a nonlinear correlation computer program. We assume the records are similar between sites due to their proximity, and therefore the signal differences are due to sample spacing or core recovery. Gaps or important sedimentation rate changes in either section will appear as significant changes in slope of the mapping function that relates one section to the other. The direction of change of the mapping function suggests which hole is missing section at a given core break. Third, the patterns and values of the signals are examined at a large scale to determine the extent of the stratigraphic discontinuity of the holes. When section is missing, the appropriate interval is added from the record of the adjacent hole. After the adjustments are made, the records are recorrelated using CORPAC to test for similarity.

\section{CORING AND RECOVERY}

The pelagic sediments of Sites $504\left(1^{\circ} 13.571^{\prime} \mathrm{N}, 83^{\circ} 43.942^{\prime} \mathrm{W}\right.$; $3460 \mathrm{~m}$ water depth) and $677\left(1^{\circ} 12.140^{\prime} \mathrm{N}, 83^{\circ} 44.220^{\prime} \mathrm{W}\right.$; 
$3461.2 \mathrm{~m}$ water depth) were recovered by hydraulic piston coring. Site 504 is located $2.7 \mathrm{~km}$ northeast of Site 677 , and Holes $677 \mathrm{~A}$ and $677 \mathrm{~B}$ are offset by approximately $30 \mathrm{~m}$. The cores have been described by the Leg 69 Shipboard Scientific Party (1983) and Leg 111 Shipboard Scientific Party (1988). The sediments consist of alternating clayey siliceous nannofossil ooze and calcareous diatom ooze, and nannofossil chalks in the deeper sections.

Piston coring is the best method available to recover complete, relatively undisturbed sediments. In brief, hydraulic pressure is used to force an inner core barrel past the coring bit, through the sediments. In 1979, when Hole 504 was drilled, DSDP used a hydraulic piston corer (HPC) with a 4.4-m-long core barrel. Recovery was good, but many gaps are apparent at core breaks and drilling disturbances are more numerous and usually larger than in the Site 677 sediments. ODP improved upon the DSDP HPC with the advanced piston corer (APC), which uses higher hydraulic pressure and has a 9.5-m-long core barrel. For hard sediments, not readily penetrated with the APC, ODP retrieves cores with the extended core barrel (XCB). The XCB is basically a rotary drill, but it has a cutting shoe that can extend beyond the core bit when coring in relatively soft sediments to prevent drilling disturbances by the bit's hydraulic jets.

In Hole $677 \mathrm{~A}$, the first 15 cores $(0-139.2 \mathrm{~m}$ below seafloor [mbsf]) were recovered using the APC. Below this depth the $\mathrm{XCB}$ was used to recover Cores 111-677A-16X through 111677A-34X. A second hole at this site, Hole 677B, was cored to a sub-bottom depth of $93.1 \mathrm{~m}$, using the APC. The cores from Hole $677 \mathrm{~B}$ were purposely staggered by approximately $2.5 \mathrm{~m}$ with respect to Hole 677A in order for core break depths in Hole 677B to correspond to mid-core depths in Hole 677A and vice versa. As will be discussed subsequently, the staggered offset between the core break depths of Holes 677A and 677B did not remain constant.

\section{LABORATORY METHODS}

Sediment samples $5 \mathrm{~cm}^{3}$ in size were obtained from Holes $677 \mathrm{~A}$ and $677 \mathrm{~B}$ at $50-\mathrm{cm}$ (approximately 10,000-yr) intervals. This sample spacing was chosen in order to match the sample spacing previously obtained by the authors in the Pliocene section of DSDP Hole 504 and to recover signals with enough character for nonlinear correlation. Each sample was dried in a $60^{\circ} \mathrm{C}$ oven overnight, and a split (approximately $1.2 \mathrm{~g}$ dry weight) was crushed in an agate mortar and stored in glass vials. Percent carbonate analysis (Table 1) was performed using a modified version of the vacuum-gasometric technique of Jones and Kaiteris (1983), with an estimated analytical precision of $\pm 0.25 \%$. Percent Si-opal (weight percent of dry samples composed of opaline silicon) determinations (Table 1) were performed using the sodium carbonate extraction molybdate blue spectrophotometric technique of Mortlock and Froelich (in press), with an estimated reproducibility of $\pm 5 \%$ of the measured value. The nonbiogenic component is calculated as

$$
100-\% \text { carbonate }-2.4(\% \mathrm{Si}-\text { opal }) \text {. }
$$

The 2.4 factor in determining the weight percent of opal accounts for the formula weight of opal with a water content of $10 \%$ (Mortlock and Froelich, in press). Percent calcium carbonate data from Hole 504 were taken from the Leg 69 barrel sheets (Shipboard Scientific Party, 1983) and supplemented with additional analyses (Table 2). Shipboard carbonate analyses were performed using the "Karbonat-Bombe" technique (Müller and Gastner, 1971).

\section{CORRELATIONS}

The key to constructing a complete stratigraphic column from double- or triple-cored sites is to (1) correlate age-equivalent horizons between holes, (2) locate differences in sedimentation rate that can be caused by stretching or squeezing of cores by the
Table 1. Percent carbonate and percent Si-opal values of samples from ODP Leg 111 Holes $677 \mathrm{~A}$ and 677B.

\begin{tabular}{|c|c|c|c|}
\hline $\begin{array}{l}\text { Core, section, } \\
\text { interval }(\mathrm{cm})\end{array}$ & $\begin{array}{l}\text { Depth }^{a} \\
\text { (mbsf) }\end{array}$ & $\begin{array}{c}\text { Calcium } \\
\text { carbonate } \\
(\%)\end{array}$ & $\begin{array}{c}\text { Si-opa } \\
(\%)\end{array}$ \\
\hline \multicolumn{4}{|l|}{$111-677 \mathrm{~A}-$} \\
\hline $1 \mathrm{H}-1,23-24$ & 0.24 & 32.75 & 5.40 \\
\hline $1 \mathrm{H}-1,73-74$ & 0.74 & 52.79 & 3.49 \\
\hline $1 \mathrm{H}-1,135-137$ & 1.37 & 45.66 & 4.81 \\
\hline $1 \mathrm{H}-2,46-48$ & 1.98 & 43.16 & 5.51 \\
\hline $1 \mathrm{H}-2,73-74$ & 2.24 & 44.95 & 5.45 \\
\hline $1 \mathrm{H}-2,123-124$ & 2.74 & 42.48 & 7.12 \\
\hline $1 \mathrm{H}-3,24-26$ & 3.26 & 44.34 & 6.83 \\
\hline $1 \mathrm{H}-3,73-74$ & 3.74 & 19.83 & 7.72 \\
\hline $1 \mathrm{H}-3,123-124$ & 4.24 & 21.49 & 7.34 \\
\hline $1 \mathrm{H}-4,23-24$ & 4.74 & 25.97 & 6.97 \\
\hline $1 \mathrm{H}-4,73-74$ & 5.24 & 31.41 & 6.98 \\
\hline $1 \mathrm{H}-4,125-127$ & 5.77 & 10.52 & 5.77 \\
\hline $2 \mathrm{H}-1,23-24$ & 6.44 & 39.88 & 5.45 \\
\hline $2 \mathrm{H}-1,50-52$ & 6.72 & 21.17 & 7.61 \\
\hline $2 \mathrm{H}-1,123-124$ & 7.44 & 26.34 & 7.55 \\
\hline $2 \mathrm{H}-2,7-9$ & 7.79 & 30.84 & 7.08 \\
\hline $2 \mathrm{H}-2,73-75$ & 8.45 & 14.75 & 4.93 \\
\hline $2 \mathrm{H}-2,123-124$ & 8.94 & 30.95 & 6.31 \\
\hline $2 \mathrm{H}-3,23-24$ & 9.44 & 52.59 & 4.28 \\
\hline $2 \mathrm{H}-3,73-74$ & 9.94 & 29.32 & 7.41 \\
\hline $2 \mathrm{H}-3,123-125$ & 10.45 & 25.36 & 8.09 \\
\hline $2 \mathrm{H}-4,9-10$ & 10.80 & 47.55 & 6.75 \\
\hline $2 \mathrm{H}-4,73-74$ & 11.44 & 40.67 & 6.82 \\
\hline $2 \mathrm{H}-4,123-124$ & 11.94 & 28.44 & 7.19 \\
\hline $2 \mathrm{H}-5,23-24$ & 12.44 & 49.73 & 4.97 \\
\hline $2 \mathrm{H}-5,73-75$ & 12.94 & 52.60 & 4.49 \\
\hline $2 \mathrm{H}-5,129-131$ & 13.51 & 38.35 & 6.29 \\
\hline $2 \mathrm{H}-6,23-24$ & 13.94 & 44.54 & 5.56 \\
\hline $2 \mathrm{H}-6,73-75$ & 14.44 & 39.32 & 5.53 \\
\hline $2 \mathrm{H}-6,125-127$ & 14.97 & 38.72 & 5.00 \\
\hline $2 \mathrm{H}-7,23-24$ & 15.44 & 51.33 & 4.24 \\
\hline $3 \mathrm{H}-1,23-24$ & 15.94 & 61.21 & 3.43 \\
\hline $3 \mathrm{H}-1,83-85$ & 16.55 & 57.93 & 3.63 \\
\hline $3 \mathrm{H}-1,123-124$ & 16.94 & 55.04 & 4.17 \\
\hline $3 \mathrm{H}-2,23-24$ & 17.44 & 37.39 & 6.30 \\
\hline $3 \mathrm{H}-2,83-84$ & 18.05 & 48.67 & 4.67 \\
\hline $3 \mathrm{H}-2,123-124$ & 18.44 & 43.43 & 5.18 \\
\hline $3 \mathrm{H}-3,23-24$ & 18.94 & 42.86 & 5.53 \\
\hline $3 \mathrm{H}-3,83-85$ & 19.55 & 52.19 & 5.23 \\
\hline $3 \mathrm{H}-3,123-124$ & 19.94 & 42.55 & 5.24 \\
\hline $3 \mathrm{H}-4,23-24$ & 20.44 & 24.09 & 7.72 \\
\hline $3 \mathrm{H}-4,83-85$ & 21.05 & 47.08 & 5.08 \\
\hline $3 \mathrm{H}-5,23-24$ & 21.94 & 23.00 & 7.08 \\
\hline $3 \mathrm{H}-5,83-85$ & 22.55 & 44.44 & 5.38 \\
\hline $3 \mathrm{H}-5,123-124$ & 22.94 & 46.98 & 5.56 \\
\hline $3 \mathrm{H}-6,23-24$ & 23.44 & 54.45 & 4.88 \\
\hline $3 \mathrm{H}-6,83-85$ & 24.05 & 45.50 & 6.18 \\
\hline $3 \mathrm{H}-6,123-124$ & 24.44 & 31.41 & 8.01 \\
\hline $3 \mathrm{H}-7,23-24$ & 24.94 & 14.97 & 9.52 \\
\hline $4 \mathrm{H}-1,23-24$ & 25.44 & 43.74 & 7.68 \\
\hline $4 \mathrm{H}-1,92-94$ & 26.14 & 42.15 & 7.79 \\
\hline $4 \mathrm{H}-1,123-124$ & 26.44 & 32.54 & 7.54 \\
\hline $4 \mathrm{H}-2,23-24$ & 26.94 & 19.03 & 11.61 \\
\hline $4 \mathrm{H}-2,89-91$ & 27.61 & 42.63 & 8.20 \\
\hline $4 \mathrm{H}-2,123-124$ & 27.94 & 61.23 & 5.57 \\
\hline $4 \mathrm{H}-3,23-24$ & 28.44 & 42.87 & 7.57 \\
\hline $4 \mathrm{H}-3,92-94$ & 29.14 & 39.93 & 9.89 \\
\hline $4 \mathrm{H}-3,123-124$ & 29.44 & 38.71 & 9.62 \\
\hline $4 \mathrm{H}-4,23-24$ & 29.94 & 35.31 & 9.66 \\
\hline $4 \mathrm{H}-4,92-94$ & 30.64 & 48.44 & 7.12 \\
\hline $4 \mathrm{H}-4,123-124$ & 30.94 & 49.76 & 7.00 \\
\hline $4 \mathrm{H}-5,23-24$ & 31.44 & 46.42 & 6.10 \\
\hline $4 \mathrm{H}-5,92-94$ & 32.14 & 49.48 & 5.48 \\
\hline $4 \mathrm{H}-5,123-124$ & 32.44 & 58.54 & 5.25 \\
\hline $4 \mathrm{H}-6,23-24$ & 32.94 & 54.38 & 8.50 \\
\hline $4 \mathrm{H}-6,92-94$ & 33.64 & 29.59 & 9.18 \\
\hline $4 \mathrm{H}-6,123-124$ & 33.94 & 30.81 & 9.19 \\
\hline $5 \mathrm{H}-1,23-24$ & 34.94 & 47.71 & 6.27 \\
\hline $5 \mathrm{H}-1,110-112$ & 35.82 & 25.18 & 9.60 \\
\hline $5 \mathrm{H}-2,23-24$ & 36.44 & 51.13 & 6.75 \\
\hline $5 \mathrm{H}-2,73-74$ & 36.94 & 35.43 & 9.31 \\
\hline $5 \mathrm{H}-2,110-112$ & 37.32 & 46.39 & 7.05 \\
\hline
\end{tabular}


Table 1 (continued).

\begin{tabular}{|c|c|c|c|}
\hline $\begin{array}{l}\text { Core, section, } \\
\text { interval }(\mathrm{cm})\end{array}$ & $\begin{array}{l}\text { Depth }^{a} \\
\text { (mbsf) }\end{array}$ & $\begin{array}{c}\text { Calcium } \\
\text { carbonate } \\
(\%)\end{array}$ & $\begin{array}{c}\text { Si-opal } \\
(\%)\end{array}$ \\
\hline \multicolumn{4}{|l|}{ 111-677A- (Cont.) } \\
\hline $5 \mathrm{H}-3,23-24$ & 37.94 & 53.82 & 6.76 \\
\hline $5 \mathrm{H}-3,75-76$ & 38.46 & 52.17 & 6.70 \\
\hline $5 \mathrm{H}-3,110-112$ & 38.82 & 42.58 & 8.47 \\
\hline $5 \mathrm{H}-4,23-24$ & 39.44 & 62.69 & 4.67 \\
\hline $5 \mathrm{H}-4,73-74$ & 39.94 & 43.57 & 8.00 \\
\hline $5 \mathrm{H}-4,110-112$ & 40.32 & 36.44 & 8.62 \\
\hline $5 \mathrm{H}-5,23-24$ & 40.94 & 52.79 & 7.03 \\
\hline $5 \mathrm{H}-5,73-74$ & 41.44 & 43.37 & 11.80 \\
\hline $5 \mathrm{H}-5,110-112$ & 41.82 & 43.04 & 9.84 \\
\hline $5 \mathrm{H}-6,23-24$ & 42.44 & 43.18 & 10.30 \\
\hline $5 \mathrm{H}-6,73-74$ & 42.94 & 51.68 & 9.51 \\
\hline $5 \mathrm{H}-6,110-112$ & 43.32 & 37.09 & 8.38 \\
\hline $5 \mathrm{H}-7,23-24$ & 43.94 & 16.80 & 14.63 \\
\hline $6 \mathrm{H}-1,23-24$ & 44.44 & 33.53 & 10.89 \\
\hline $6 \mathrm{H}-1,73-74$ & 44.94 & 54.14 & 5.71 \\
\hline $6 \mathrm{H}-1,107-109$ & 45.29 & 54.04 & 8.23 \\
\hline $6 \mathrm{H}-2,24-26$ & 45.96 & 56.95 & 5.41 \\
\hline $6 \mathrm{H}-2,73-74$ & 46.44 & 48.46 & 6.10 \\
\hline $6 \mathrm{H}-2,123-124$ & 46.94 & 33.97 & 8.85 \\
\hline $6 \mathrm{H}-3,12-14$ & 47.34 & 54.60 & 5.90 \\
\hline $6 \mathrm{H}-3,73-74$ & 47.94 & 55.76 & 6.87 \\
\hline $6 \mathrm{H}-4,23-24$ & 48.94 & 28.07 & 8.46 \\
\hline $6 \mathrm{H}-4,73-74$ & 49.44 & 44.28 & 6.79 \\
\hline $6 \mathrm{H}-4,124-126$ & 49.96 & 49.31 & 6.77 \\
\hline $6 \mathrm{H}-5,23-24$ & 50.44 & 32.70 & 8.96 \\
\hline $6 \mathrm{H}-5,73-74$ & 50.94 & 46.76 & 7.46 \\
\hline $6 \mathrm{H}-5,133-135$ & 51.55 & 48.54 & 8.02 \\
\hline $6 \mathrm{H}-6,23-24$ & 51.94 & 48.56 & 6.24 \\
\hline $6 \mathrm{H}-6,77-79$ & 52.49 & 28.65 & 9.47 \\
\hline $6 \mathrm{H}-6,123-124$ & 52.94 & 51.28 & 4.93 \\
\hline $6 \mathrm{H}-7,7-9$ & 53.29 & 59.59 & 4.52 \\
\hline $7 \mathrm{H}-1,23-24$ & 53.94 & 60.52 & 5.41 \\
\hline $7 \mathrm{H}-1,64-66$ & 54.36 & 60.90 & 5.39 \\
\hline $7 \mathrm{H}-1,123-124$ & 54.94 & 57.20 & 5.71 \\
\hline $7 \mathrm{H}-2,23-24$ & 55.44 & 40.79 & 7.32 \\
\hline $7 \mathrm{H}-2,64-66$ & 55.86 & 31.23 & 8.85 \\
\hline $7 \mathrm{H}-2,123-124$ & 56.44 & 50.77 & 7.01 \\
\hline $7 \mathrm{H}-3,23-24$ & 56.94 & 40.10 & 10.30 \\
\hline $7 \mathrm{H}-3,64-66$ & 57.36 & 37.49 & 6.99 \\
\hline $7 \mathrm{H}-3,123-124$ & 57.94 & 48.95 & 6.37 \\
\hline $7 \mathrm{H}-4,23-24$ & 58.44 & 53.61 & 7.71 \\
\hline $7 \mathrm{H}-4,64-66$ & 58.86 & 47.57 & 7.03 \\
\hline $7 \mathrm{H}-4,123-124$ & 59.44 & 46.17 & 8.17 \\
\hline $7 \mathrm{H}-5,23-24$ & 59.94 & 47.31 & 8.14 \\
\hline $7 \mathrm{H}-5,64-66$ & 60.36 & 29.72 & 8.56 \\
\hline $7 \mathrm{H}-5,123-124$ & 60.94 & 27.46 & 8.84 \\
\hline $7 \mathrm{H}-6,23-24$ & 61.44 & 49.80 & 6.02 \\
\hline $7 \mathrm{H}-6,64-66$ & 61.86 & 23.03 & 9.67 \\
\hline $7 \mathrm{H}-6,123-124$ & 62.44 & 21.66 & 9.32 \\
\hline $8 \mathrm{H}-1,23-24$ & 63.44 & 51.87 & 6.07 \\
\hline $8 \mathrm{H}-1,84-86$ & 64.06 & 30.28 & 9.61 \\
\hline $8 \mathrm{H}-1,123-124$ & 64.44 & 38.76 & 8.35 \\
\hline $8 \mathrm{H}-2,23-24$ & 64.94 & 19.24 & 11.56 \\
\hline $8 \mathrm{H}-2,84-86$ & 65.56 & 7.82 & 12.54 \\
\hline $8 \mathrm{H}-2,123-124$ & 65.94 & 15.85 & 11.31 \\
\hline $8 \mathrm{H}-3,23-24$ & 66.44 & 11.34 & 13.26 \\
\hline $8 \mathrm{H}-3,84-86$ & 67.06 & 8.67 & 11.08 \\
\hline $8 \mathrm{H}-3,123-124$ & 67.44 & 9.01 & 12.38 \\
\hline $8 \mathrm{H}-4,23-24$ & 67.94 & 29.87 & 9.79 \\
\hline $8 \mathrm{H}-4,84-86$ & 68.56 & 43.01 & 7.89 \\
\hline $8 \mathrm{H}-4,123-124$ & 68.94 & 21.72 & 9.29 \\
\hline $8 \mathrm{H}-5,23-24$ & 69.44 & 10.67 & 11.35 \\
\hline $8 \mathrm{H}-5,84-86$ & 60.06 & 51.51 & 9.73 \\
\hline $8 \mathrm{H}-5,123-124$ & 70.44 & 16.39 & 12.02 \\
\hline $8 \mathrm{H}-6,23-24$ & 70.94 & 20.47 & 9.57 \\
\hline $8 \mathrm{H}-6,84-86$ & 71.56 & 14.57 & 12.29 \\
\hline $8 \mathrm{H}-6,123-124$ & 71.94 & 18.07 & 11.35 \\
\hline $8 \mathrm{H}-7,23-24$ & 72.44 & 13.66 & 13.39 \\
\hline $9 \mathrm{H}-1,22-24$ & 72.94 & 17.68 & 11.03 \\
\hline $9 H-1,61-63$ & 73.33 & 26.69 & 10.42 \\
\hline $9 \mathrm{H}-1,124-126$ & 73.96 & 32.36 & 8.53 \\
\hline $9 \mathrm{H}-2,21-23$ & 74.43 & 20.66 & 10.78 \\
\hline $9 \mathrm{H}-2,61-63$ & 74.83 & 14.49 & 13.16 \\
\hline $9 \mathrm{H}-2,124-126$ & 75.46 & 30.99 & 10.80 \\
\hline
\end{tabular}

Table 1 (continued).

\begin{tabular}{|c|c|c|c|}
\hline $\begin{array}{l}\text { Core, section, } \\
\text { interval }(\mathrm{cm})\end{array}$ & $\begin{array}{l}\text { Depth }{ }^{a} \\
\text { (mbsf) }\end{array}$ & $\begin{array}{l}\text { Calcium } \\
\text { carbonate } \\
(\%)\end{array}$ & $\begin{array}{c}\text { Si-opal } \\
(\%)\end{array}$ \\
\hline \multicolumn{4}{|l|}{ 111-677A- (Cont.) } \\
\hline $9 \mathrm{H}-3,22-23$ & 75.93 & 24.48 & 12.09 \\
\hline $9 \mathrm{H}-3,60-62$ & 76.32 & 29.84 & 11.34 \\
\hline $9 \mathrm{H}-3,104-105$ & 76.75 & 23.99 & \\
\hline $9 \mathrm{H}-4,22-23$ & 77.43 & 46.00 & 8.85 \\
\hline $9 \mathrm{H}-4,60-62$ & 77.82 & 39.99 & 10.04 \\
\hline $9 \mathrm{H}-4,125-126$ & 78.46 & 35.53 & 11.58 \\
\hline $9 \mathrm{H}-5,22-23$ & 78.93 & 34.43 & 14.43 \\
\hline $9 \mathrm{H}-5,62-64$ & 79.34 & 33.06 & 13.72 \\
\hline $9 \mathrm{H}-5,125-126$ & 79.96 & 31.47 & 15.68 \\
\hline $9 \mathrm{H}-6,22-23$ & 80.43 & 22.34 & 17.42 \\
\hline $9 \mathrm{H}-6,63-65$ & 80.85 & 33.40 & 11.15 \\
\hline $9 \mathrm{H}-6,116-117$ & 81.37 & 44.11 & 10.67 \\
\hline $10 \mathrm{H}-1,22-23$ & 82.43 & 27.62 & \\
\hline $10 \mathrm{H}-1,62-64$ & 82.84 & 22.15 & 10.30 \\
\hline $10 \mathrm{H}-1,122-124$ & 83.44 & 27.29 & 8.92 \\
\hline $10 \mathrm{H}-2,22-23$ & 83.93 & 25.51 & 8.06 \\
\hline $10 \mathrm{H}-2,61-63$ & 84.33 & 13.83 & 11.20 \\
\hline $10 \mathrm{H}-2,122-123$ & 84.94 & 22.51 & 12.67 \\
\hline $10 \mathrm{H}-3,21-22$ & 85.42 & 29.47 & 9.13 \\
\hline $10 \mathrm{H}-3,62-64$ & 85.84 & 10.34 & 11.56 \\
\hline $10 \mathrm{H}-3,123-124$ & 86.44 & 30.27 & 10.01 \\
\hline $10 \mathrm{H}-4,22-23$ & 86.93 & 38.12 & 8.31 \\
\hline $10 \mathrm{H}-4,61-63$ & 87.33 & 53.10 & 5.46 \\
\hline $10 \mathrm{H}-4,123-124$ & 87.94 & 46.12 & 6.60 \\
\hline $10 \mathrm{H}-5,22-23$ & 88.43 & 46.85 & 5.31 \\
\hline $10 \mathrm{H}-5,62-64$ & 88.84 & 49.20 & 7.51 \\
\hline $10 \mathrm{H}-5,123-124$ & 89.44 & 35.68 & 9.16 \\
\hline $10 \mathrm{H}-6,23-24$ & 89.94 & 32.82 & 12.61 \\
\hline $10 \mathrm{H}-6,62-64$ & 90.34 & 34.75 & 9.94 \\
\hline $10 \mathrm{H}-6,123-124$ & 90.94 & 37.16 & 9.37 \\
\hline $10 \mathrm{H}-7,22-23$ & 91.43 & 52.76 & 5.38 \\
\hline $11 \mathrm{H}-1,23-24$ & 91.94 & 45.06 & 6.94 \\
\hline $11 \mathrm{H}-1,84-86$ & 92.56 & 38.33 & 8.30 \\
\hline $11 \mathrm{H}-1,123-124$ & 92.94 & 50.18 & 6.33 \\
\hline $11 \mathrm{H}-2,23-24$ & 93.44 & 46.10 & 7,44 \\
\hline $11 \mathrm{H}-2,84-86$ & 94.06 & 39.90 & 7.24 \\
\hline $11 \mathrm{H}-2,123-124$ & 94.44 & 46.43 & 8.20 \\
\hline $11 \mathrm{H}-3,23-24$ & 94.94 & 50.09 & 7.37 \\
\hline $11 \mathrm{H}-3,84-86$ & 95.56 & 47.31 & 7.42 \\
\hline $11 \mathrm{H}-3,123-124$ & 95.94 & 54.40 & 6.65 \\
\hline $11 \mathrm{H}-4,23-24$ & 96.44 & 53.80 & 7.25 \\
\hline $11 \mathrm{H}-4,84-86$ & 97.06 & 49.46 & 8.29 \\
\hline $11 \mathrm{H}-4,123-124$ & 97.44 & 56.51 & 5.61 \\
\hline $11 \mathrm{H}-5,23-24$ & 97.94 & 66.00 & 5.40 \\
\hline $11 \mathrm{H}-5,84-86$ & 98.56 & 51.99 & 8.39 \\
\hline $11 \mathrm{H}-5,123-124$ & 98.94 & 35.99 & 11.16 \\
\hline $11 \mathrm{H}-6,23-24$ & 99.44 & 36.47 & 10.20 \\
\hline $11 \mathrm{H}-6,84-86$ & 100.06 & 43.48 & 7.62 \\
\hline $11 \mathrm{H}-6,123-124$ & 100.44 & 52.71 & 6.49 \\
\hline $11 \mathrm{H}-7,23-24$ & 100.94 & 65.85 & 5.12 \\
\hline $12 \mathrm{H}-1,23-24$ & 101.44 & 59.80 & 5.23 \\
\hline $12 \mathrm{H}-1,84-86$ & 102.06 & 43.91 & 8.12 \\
\hline $12 \mathrm{H}-1,123-124$ & 102.44 & 37.04 & 10.04 \\
\hline $12 \mathrm{H}-2,23-24$ & 102.94 & 10.84 & 15.33 \\
\hline $12 \mathrm{H}-2,84-86$ & 103.56 & 33.71 & 8.41 \\
\hline $12 \mathrm{H}-2,124-125$ & 103.95 & 37.49 & 9.13 \\
\hline $12 \mathrm{H}-3,23-24$ & 104.44 & 34.92 & 9.54 \\
\hline $12 \mathrm{H}-3,84-86$ & 105.06 & 33.12 & 9.74 \\
\hline $12 \mathrm{H}-3,123-124$ & 105.44 & 56.36 & 5.98 \\
\hline $12 \mathrm{H}-4,23-24$ & 105.94 & 39.93 & 11.24 \\
\hline $12 \mathrm{H}-4,84-86$ & 106.56 & 50.97 & 6.83 \\
\hline $12 \mathrm{H}-5,23-24$ & 107.44 & 51.56 & 8.13 \\
\hline $12 \mathrm{H}-5,84-86$ & 108.06 & 38.87 & 10.23 \\
\hline $12 \mathrm{H}-5,123-124$ & 108.44 & 24.37 & 12.13 \\
\hline $12 \mathrm{H}-6,23-24$ & 108.94 & 44.79 & 7.48 \\
\hline $12 \mathrm{H}-6,84-86$ & 109.56 & 39.03 & 9.79 \\
\hline $12 \mathrm{H}-6,123-124$ & 109.94 & 52.77 & 7.49 \\
\hline $12 \mathrm{H}-7,23-24$ & 110.44 & 36.44 & 10.48 \\
\hline $13 \mathrm{H}-1,22-23$ & 110.93 & 38.42 & 13.39 \\
\hline $13 \mathrm{H}-1,84-86$ & 111.56 & 43.37 & 9.81 \\
\hline $13 \mathrm{H}-1,123-124$ & 111.94 & 50.86 & 9.08 \\
\hline $13 \mathrm{H}-2,22-23$ & 112.43 & 54.87 & 8.10 \\
\hline $13 \mathrm{H}-2,84-86$ & 113.06 & 57.77 & 7.30 \\
\hline $13 \mathrm{H}-2,123-124$ & 113.44 & 52.82 & 10.58 \\
\hline
\end{tabular}


Table 1 (continued).

\begin{tabular}{|c|c|c|c|}
\hline $\begin{array}{l}\text { Core, section, } \\
\text { interval }(\mathrm{cm})\end{array}$ & $\begin{array}{l}\text { Depth }^{a} \\
\text { (mbsf) }\end{array}$ & $\begin{array}{c}\text { Calcium } \\
\text { carbonate } \\
(\%)\end{array}$ & $\begin{array}{c}\text { Si-opal } \\
(\%)\end{array}$ \\
\hline \multicolumn{4}{|l|}{ 111-677A- (Cont.) } \\
\hline $13 \mathrm{H}-3,23-24$ & 113.94 & 50.34 & 7.54 \\
\hline $13 \mathrm{H}-3,84-86$ & 114.56 & 45.39 & 7.39 \\
\hline $13 \mathrm{H}-3,123-124$ & 114.94 & 59.57 & 6.08 \\
\hline $13 \mathrm{H}-4,23-24$ & 115.44 & 56.60 & 6.69 \\
\hline $13 \mathrm{H}-4,84-86$ & 110.06 & 35.68 & 12.31 \\
\hline $13 \mathrm{H}-4,124-125$ & 116.45 & 63.51 & 5.26 \\
\hline $13 \mathrm{H}-5,23-24$ & 116.94 & 61.73 & 5.66 \\
\hline $13 \mathrm{H}-5,84-86$ & 117.56 & 56.62 & 7.42 \\
\hline $13 \mathrm{H}-5,123-124$ & 117.94 & 45.55 & 8.61 \\
\hline $13 \mathrm{H}-6,23-24$ & 118.44 & 53.33 & 6.87 \\
\hline $13 \mathrm{H}-6,84-86$ & 119.06 & 53.52 & 6.69 \\
\hline $13 \mathrm{H}-6,123-124$ & 119.44 & 38.88 & 9.78 \\
\hline $13 \mathrm{H}-7,23-24$ & 119.94 & 42.15 & 10.63 \\
\hline $14 \mathrm{H}-1,23-24$ & 120.44 & 42.92 & 8.83 \\
\hline $14 \mathrm{H}-1,84-86$ & 121.06 & 47.88 & 6.84 \\
\hline $14 \mathrm{H}-1,123-124$ & 121.44 & 44.62 & 9.25 \\
\hline $14 \mathrm{H}-2,23-24$ & 121.94 & 54.28 & 7.29 \\
\hline $14 \mathrm{H}-2,84-86$ & 122.56 & 37.88 & 11.10 \\
\hline $14 \mathrm{H}-2,123-124$ & 122.94 & 46.71 & 9.63 \\
\hline $14 \mathrm{H}-3,23-24$ & 123.44 & 51.54 & 8.57 \\
\hline $14 \mathrm{H}-3,84-86$ & 124.06 & 43.51 & 10.43 \\
\hline $14 \mathrm{H}-3,123-124$ & 124.44 & 52.78 & 9.11 \\
\hline $14 \mathrm{H}-4,23-24$ & 124.94 & 54.47 & 7.77 \\
\hline $14 \mathrm{H}-4,84-86$ & 125.56 & 44.96 & 8.54 \\
\hline $14 \mathrm{H}-4,123-124$ & 125.94 & 51.40 & 6.44 \\
\hline $14 \mathrm{H}-5,23-24$ & 126.44 & 31.78 & 9.97 \\
\hline $14 \mathrm{H}-5,84-86$ & 127.06 & 37.04 & 6.82 \\
\hline $14 \mathrm{H}-5,123-124$ & 127.44 & 31.68 & 8.66 \\
\hline $14 \mathrm{H}-6,23-24$ & 127.94 & 41.43 & 7.72 \\
\hline $14 \mathrm{H}-6,84-86$ & 128.56 & 45.35 & 7.47 \\
\hline $14 \mathrm{H}-6,123-124$ & 128.94 & 51.20 & 6.60 \\
\hline $14 \mathrm{H}-7,23-24$ & 129.44 & 35.30 & 7.49 \\
\hline $15 \mathrm{H}-1,23-24$ & 129.94 & 42.51 & 7.49 \\
\hline $15 \mathrm{H}-1,84-86$ & 130.56 & 46.25 & 7.22 \\
\hline $15 \mathrm{H}-1,123-124$ & 130.94 & 40.08 & 8.25 \\
\hline $15 \mathrm{H}-2,23-24$ & 131.44 & 33.50 & 7.39 \\
\hline $15 \mathrm{H}-2,84-86$ & 132.06 & 51.34 & 6.43 \\
\hline $15 \mathrm{H}-2,123-124$ & 132.44 & 49.15 & 6.49 \\
\hline $15 \mathrm{H}-3,23-24$ & 132.94 & 56.54 & 5.56 \\
\hline $15 \mathrm{H}-3,84-86$ & 133.56 & 42.87 & 8.57 \\
\hline $15 \mathrm{H}-3,123-124$ & 133.94 & 55.13 & 7.54 \\
\hline $15 \mathrm{H}-4,23-24$ & 134.44 & 50.87 & 6.30 \\
\hline $15 \mathrm{H}-4,84-86$ & 135.06 & 45.71 & 7.31 \\
\hline $15 \mathrm{H}-5,23-24$ & 135.94 & 53.98 & 7.20 \\
\hline $15 \mathrm{H}-5,84-86$ & 136.56 & 48.74 & 7.04 \\
\hline $15 \mathrm{H}-5,123-124$ & 136.94 & 38.56 & 6.83 \\
\hline $15 \mathrm{H}-6,23-24$ & 137.44 & 54.25 & 5.64 \\
\hline $15 \mathrm{H}-6,84-86$ & 138.06 & 43.32 & 9.15 \\
\hline $15 \mathrm{H}-6,123-124$ & 138.44 & 50.98 & 7.27 \\
\hline $15 \mathrm{H}-7,23-24$ & 138.94 & 32.38 & 7.64 \\
\hline $16 X-1,23-24$ & 139.44 & 48.80 & 6.82 \\
\hline $16 \mathrm{X}-1,127-128$ & 139.98 & 49.71 & 6.99 \\
\hline $16 X-2,23-24$ & 140.41 & 64.74 & 6.20 \\
\hline $16 \mathrm{X}-2,118-119$ & 140.74 & 60.68 & 5.86 \\
\hline $16 \mathrm{X}-3,119-120$ & 141.16 & 56.61 & 6.31 \\
\hline $16 X-4,23-24$ & 141.67 & 58.92 & 5.51 \\
\hline $16 \times-4,118-119$ & 142.62 & 41.21 & 7.79 \\
\hline $16 X-5,23-24$ & 143.17 & 59.39 & 4.64 \\
\hline $16 X-5,118-119$ & 144.12 & 60.36 & 5.16 \\
\hline $17 X-1,23-24$ & 144.44 & 55.79 & 5.31 \\
\hline $17 X-1,84-85$ & 145.05 & 55.78 & 4.46 \\
\hline $17 X-1,123-124$ & 145.44 & 62.23 & 4.89 \\
\hline $17 X-2,23-24$ & 145.94 & 62.47 & 5.54 \\
\hline $17 X-2,84-85$ & 146.55 & 62.57 & 4.42 \\
\hline $17 X-2,123-124$ & 146.94 & 62.86 & 4.01 \\
\hline $17 X-3,23-24$ & 147.44 & 57.95 & 5.24 \\
\hline $17 X-3,84-85$ & 148.05 & 57.28 & 4.50 \\
\hline $17 X-3,123-124$ & 148.44 & 61.20 & 4.79 \\
\hline $17 X-4,23-24$ & 148.94 & 55.66 & 6.65 \\
\hline $17 X-4,84-85$ & 149.55 & 67.38 & 4.38 \\
\hline $17 X-4,123-124$ & 149.94 & 65.13 & 4.55 \\
\hline $17 X-5,23-24$ & 150,44 & 75.08 & 3.04 \\
\hline $17 X-5,84-85$ & 151.05 & 62.44 & 4.09 \\
\hline $17 X-5,123-124$ & 151.44 & 60.73 & 4.08 \\
\hline
\end{tabular}

Table 1 (continued).

\begin{tabular}{|c|c|c|c|}
\hline $\begin{array}{l}\text { Core, section, } \\
\text { interval }(\mathrm{cm})\end{array}$ & $\begin{array}{l}\text { Depth }{ }^{a} \\
\text { (mbsf) }\end{array}$ & $\begin{array}{c}\text { Calcium } \\
\text { carbonate } \\
(\%)\end{array}$ & $\begin{array}{l}\text { Si-opal } \\
(\%)\end{array}$ \\
\hline \multicolumn{4}{|l|}{ 111-677A- (Cont.) } \\
\hline $18 \mathrm{X}-1,23-24$ & 154.04 & 69.34 & 3.26 \\
\hline $18 X-1,83-84$ & 154.64 & 66.85 & 3.39 \\
\hline $18 X-1,122-123$ & 155.03 & 52.11 & 4.49 \\
\hline $18 X-2,23-24$ & 155.54 & 49.73 & 5.71 \\
\hline $18 X-2,84-85$ & 156.15 & 62.21 & 4.02 \\
\hline $18 X-2,123-124$ & 156.54 & 56.31 & 4.75 \\
\hline $18 X-3,23-24$ & 157.04 & 54.91 & 5.84 \\
\hline $18 \mathrm{X}-3,84-85$ & 157.65 & 56.79 & 4.77 \\
\hline $18 X-4,23-24$ & 158.54 & 68.90 & 3.83 \\
\hline $18 \times-4,84-85$ & 159.15 & 58.33 & 3.85 \\
\hline $18 X-4,123-124$ & 159.54 & 51.49 & 6.45 \\
\hline $19 X-1,23-24$ & 163.74 & 62.17 & 7.81 \\
\hline $19 X-1,83-84$ & 164.34 & 54.15 & 6.08 \\
\hline $19 X-1,123-124$ & 164.74 & 63.27 & 4.79 \\
\hline $19 X-2,23-24$ & 165.24 & 47.88 & 6.51 \\
\hline $19 X-2,83-84$ & 165.84 & 60.45 & 6.46 \\
\hline $19 X-2,123-124$ & 166.24 & 60.82 & 5.42 \\
\hline $19 X-3,23-24$ & 166.74 & 61.29 & 4.17 \\
\hline $19 X-3,84-85$ & 167.35 & 63.96 & 4.35 \\
\hline $19 X-3,123-124$ & 167.74 & 71.65 & 2.91 \\
\hline $19 X-4,23-24$ & 168.24 & 66.28 & 3.34 \\
\hline $19 X-4,83-84$ & 168.84 & 68.42 & 4.42 \\
\hline $19 X-4,123-124$ & 169.24 & 66.28 & 3.19 \\
\hline $19 X-5,23-24$ & 169.74 & 66.53 & 3.35 \\
\hline $19 X-5,83-84$ & 170.34 & 65.10 & 3.10 \\
\hline $19 X-5,123-124$ & 170.74 & 70.58 & 2.96 \\
\hline $19 X-6,23-24$ & 171.24 & 67.82 & 3.32 \\
\hline $19 X-6,83-84$ & 171.84 & 70.43 & 3.33 \\
\hline $19 X-6,123-124$ & 172.24 & 66.25 & 4.00 \\
\hline
\end{tabular}

111-677B-

$\begin{array}{lrll}1 \mathrm{H}-1,33-34 & 0.34 & 48.00 & 5.03 \\ 1 \mathrm{H}-1,83-84 & 0.84 & 46.62 & 4.51 \\ 1 \mathrm{H}-1,133-134 & 1.34 & 42.68 & 6.25 \\ 1 \mathrm{H}-2,33-34 & 1.84 & 43.80 & 5.90 \\ 1 \mathrm{H}-2,83-84 & 2.34 & 41.79 & 7.88 \\ 1 \mathrm{H}-2,133-134 & 2.84 & 40.76 & 7.57 \\ 1 \mathrm{H}-3,33-34 & 3.34 & 13.46 & 8.34 \\ 1 \mathrm{H}-3,83-84 & 3.84 & 23.59 & 6.35 \\ 1 \mathrm{H}-3,133-134 & 4.34 & 28.05 & 7.68 \\ 1 \mathrm{H}-4,33-34 & 4.84 & 19.51 & 8.26 \\ 1 \mathrm{H}-4,83-84 & 5.34 & 28.50 & 7.01 \\ 1 \mathrm{H}-4,133-134 & 5.84 & 44.63 & 6.01 \\ 1 \mathrm{H}-5,33-34 & 6.34 & 35.54 & 7.29 \\ 1 \mathrm{H}-5,83-84 & 6.84 & 36.93 & 6.72 \\ 1 \mathrm{H}-5,133-134 & 7.34 & 40.63 & 6.79 \\ 2 \mathrm{H}-1,33-34 & 7.94 & 19.60 & 7.92 \\ 2 \mathrm{H}-1,83-84 & 8.44 & 30.58 & 7.28 \\ 2 \mathrm{H}-1,133-134 & 8.94 & 26.14 & 6.21 \\ 2 \mathrm{H}-2,33-34 & 9.44 & 24.62 & 7.84 \\ 2 \mathrm{H}-2,83-84 & 9.94 & 48.19 & 4.57 \\ 2 \mathrm{H}-2,133-134 & 10.44 & 46.75 & 5.50 \\ 2 \mathrm{H}-3,33-34 & 10.94 & 24.81 & 6.92 \\ 2 \mathrm{H}-3,83-84 & 11.44 & 52.41 & 6.20 \\ 2 \mathrm{H}-3,133-134 & 11.94 & 44.45 & 5.43 \\ 2 \mathrm{H}-4,33-34 & 12.44 & 47.74 & 5.60 \\ 2 \mathrm{H}-4,83-84 & 12.94 & 34.57 & 6.77 \\ 2 \mathrm{H}-4,133-134 & 13.44 & 49.82 & 4.94 \\ 2 \mathrm{H}-5,33-34 & 13.94 & 50.26 & 4.65 \\ 2 \mathrm{H}-5,83-84 & 14.44 & 40.50 & 6.31 \\ 2 \mathrm{H}-5,133-134 & 14.94 & 41.47 & 6.07 \\ 2 \mathrm{H}-6,33-34 & 15.44 & 46.68 & 5.20 \\ 2 \mathrm{H}-6,83-84 & 15.94 & 44.76 & 5.64 \\ 2 \mathrm{H}-6,133-134 & 16.44 & 49.80 & 4.57 \\ 3 \mathrm{H}-1,33-34 & 17.44 & 47.34 & 4.83 \\ 3 \mathrm{H}-1,83-84 & 17.94 & 44.18 & 5.22 \\ 3 \mathrm{H}-1,133-134 & 18.44 & 45.95 & 5.33 \\ 3 \mathrm{H}-2,33-34 & 18.94 & 52.57 & 5.25 \\ 3 \mathrm{H}-2,83-84 & 19.44 & 38.40 & 6.27 \\ 3 \mathrm{H}-2,133-134 & 19.94 & 27.93 & 7.66 \\ 3 \mathrm{H}-3,33-34 & 20.44 & 42.63 & 5.91 \\ 3 \mathrm{H}-3,83-84 & 20.94 & 27.56 & 8.51 \\ 3 \mathrm{H}-3,133-134 & 21.44 & 25.57 & 7.60\end{array}$


Table 1 (continued).

\begin{tabular}{|c|c|c|c|}
\hline $\begin{array}{l}\text { Core, section, } \\
\text { interval }(\mathrm{cm})\end{array}$ & $\begin{array}{l}\text { Depth }^{a} \\
\text { (mbsf) }\end{array}$ & $\begin{array}{c}\text { Calcium } \\
\text { carbonate } \\
(\%)\end{array}$ & $\begin{array}{l}\text { Si-opal } \\
(\%)\end{array}$ \\
\hline \multicolumn{4}{|l|}{ 111-677B- (Cont.) } \\
\hline $3 \mathrm{H}-4,83-84$ & 22.44 & 46.20 & 5.66 \\
\hline $3 \mathrm{H}-5,33-34$ & 23.44 & 50.59 & 5.32 \\
\hline $3 \mathrm{H}-5,83-84$ & 23.94 & 23.74 & 8.35 \\
\hline $3 \mathrm{H}-5,133-134$ & 24.44 & 24.79 & 7.47 \\
\hline $3 \mathrm{H}-6,33-34$ & 24.94 & 37.85 & 5.53 \\
\hline $3 \mathrm{H}-6,83-84$ & 25.44 & 50.78 & 7.95 \\
\hline $3 \mathrm{H}-6,133-134$ & 25.94 & 39.60 & 6.55 \\
\hline $3 \mathrm{H}-7,33-34$ & 26.44 & 50.38 & 7.56 \\
\hline $4 \mathrm{H}-1,33-34$ & 26.94 & 49.20 & 7.27 \\
\hline $4 \mathrm{H}-1,83-84$ & 27.44 & 46.91 & 6.50 \\
\hline $4 \mathrm{H}-1,133-134$ & 27.94 & 42.30 & 8.21 \\
\hline $4 \mathrm{H}-2,33-34$ & 28.44 & 45.90 & 8.35 \\
\hline $4 \mathrm{H}-2,83-84$ & 28.94 & 23.02 & 11.13 \\
\hline $4 \mathrm{H}-2,133-134$ & 29.44 & 51.09 & 6.76 \\
\hline $4 \mathrm{H}-3,33-34$ & 29.94 & 46.53 & 7.47 \\
\hline $4 \mathrm{H}-3,83-84$ & 30.44 & 50.70 & 6.36 \\
\hline $4 \mathrm{H}-3,133-134$ & 30.94 & 44.55 & 5.77 \\
\hline $4 \mathrm{H}-4,83-84$ & 31.94 & 53.99 & 6.17 \\
\hline $4 \mathrm{H}-4,133-134$ & 32.44 & 46.89 & 6.36 \\
\hline $4 \mathrm{H}-5,33-34$ & 32.94 & 29.25 & 8.57 \\
\hline $4 \mathrm{H}-5,83-84$ & 33.44 & 29.24 & 8.12 \\
\hline $4 \mathrm{H}-5,133-134$ & 33.94 & 53.22 & 6.23 \\
\hline $4 \mathrm{H}-6,33-34$ & 34.44 & 42.70 & 6.73 \\
\hline $4 \mathrm{H}-6,83-84$ & 34.94 & 47.39 & 6.11 \\
\hline $4 \mathrm{H}-6,133-134$ & 35.44 & 47.19 & 7.05 \\
\hline $4 \mathrm{H}-7,31-32$ & 35.92 & 53.29 & 6.62 \\
\hline $5 \mathrm{H}-1,33-34$ & 36.44 & 44.88 & 7.86 \\
\hline $5 \mathrm{H}-1,83-84$ & 36.94 & 63.58 & 5.40 \\
\hline $5 \mathrm{H}-1,133-134$ & 37.44 & 44.36 & 7.24 \\
\hline $5 \mathrm{H}-2,33-34$ & 37.94 & 30.47 & 10.92 \\
\hline $5 \mathrm{H}-2,83-84$ & 38.44 & 51.60 & 6.93 \\
\hline $5 \mathrm{H}-2,133-134$ & 38.94 & 42.25 & 9.79 \\
\hline $5 \mathrm{H}-3,33-34$ & 39.44 & 40.16 & 8.49 \\
\hline $5 \mathrm{H}-3,83-84$ & 39.94 & 35.41 & 10.52 \\
\hline $5 \mathrm{H}-3,133-134$ & 40.44 & 52.34 & 9.38 \\
\hline $5 \mathrm{H}-4,83-84$ & 41.44 & 30.16 & 11.00 \\
\hline $5 \mathrm{H}-4,133-134$ & 41.94 & 27.65 & 10.59 \\
\hline $5 \mathrm{H}-5,33-34$ & 42.44 & 36.45 & 8.82 \\
\hline $5 \mathrm{H}-5,83-84$ & 42.94 & 44.56 & 6.51 \\
\hline $5 \mathrm{H}-5,133-134$ & 43.44 & 51.96 & 7.98 \\
\hline $5 \mathrm{H}-6,33-34$ & 43.94 & 58.10 & 6.11 \\
\hline $5 \mathrm{H}-6,83-84$ & 44.44 & 57.08 & 5.21 \\
\hline $5 \mathrm{H}-6,133-134$ & 44.94 & 49.25 & 6.08 \\
\hline $5 \mathrm{H}-7,33-34$ & 45.44 & 46.91 & 6.34 \\
\hline $6 \mathrm{H}-1,33-34$ & 45.94 & 57.01 & 4.52 \\
\hline $6 \mathrm{H}-1,83-84$ & 46.44 & 27.60 & 8.06 \\
\hline $6 \mathrm{H}-1,133-134$ & 46.94 & 36.68 & 8.27 \\
\hline $6 \mathrm{H}-2,33-34$ & 47.44 & 49.11 & 6.37 \\
\hline $6 \mathrm{H}-2,83-84$ & 47.94 & 32.74 & 8.19 \\
\hline $6 \mathrm{H}-2,133-134$ & 48.44 & 48.23 & 6.62 \\
\hline $6 \mathrm{H}-3,33-34$ & 48.94 & 47.70 & 7.51 \\
\hline $6 \mathrm{H}-3,83-84$ & 49.44 & 39.47 & 5.68 \\
\hline $6 \mathrm{H}-3,133-134$ & 49.94 & 47.35 & 6.16 \\
\hline $6 \mathrm{H}-4,83-84$ & 50.94 & 37.83 & 7.49 \\
\hline $6 \mathrm{H}-5,33-34$ & 51.94 & 36.25 & 8.56 \\
\hline $6 \mathrm{H}-5,83-84$ & 52.44 & 42.75 & 8.56 \\
\hline $6 \mathrm{H}-5,133-134$ & 52.94 & 58.18 & 6.04 \\
\hline $6 \mathrm{H}-6,33-34$ & 53.44 & 56.79 & 6.16 \\
\hline $6 \mathrm{H}-6,83-84$ & 53.94 & 35.74 & 7.29 \\
\hline $6 \mathrm{H}-6,133-134$ & 54.44 & 50.51 & 6.90 \\
\hline $6 \mathrm{H}-7,33-34$ & 54.94 & 54.44 & 6.23 \\
\hline $7 \mathrm{H}-1,33-34$ & 55.44 & 59.36 & 5.23 \\
\hline $7 \mathrm{H}-1,83-84$ & 55.94 & 42.51 & 9.89 \\
\hline $7 \mathrm{H}-1,133-134$ & 56.44 & 39.82 & 6.51 \\
\hline $7 \mathrm{H}-2,33-34$ & 56.94 & 49.20 & 6.59 \\
\hline $7 \mathrm{H}-2,83-84$ & 57.44 & 48.56 & 8.64 \\
\hline $7 \mathrm{H}-2,133-134$ & 57.94 & 48.97 & 6.92 \\
\hline $7 \mathrm{H}-3,33-34$ & 58.44 & 28.41 & 9.74 \\
\hline $7 \mathrm{H}-3,83-84$ & 58.94 & 52.62 & 7.61 \\
\hline $7 \mathrm{H}-3,133-134$ & 59.44 & 36.95 & 7.84 \\
\hline $7 \mathrm{H}-4,83-84$ & 60.44 & 31.19 & 8.84 \\
\hline $7 \mathrm{H}-4,133-134$ & 60.94 & 29.74 & 7.52 \\
\hline $7 \mathrm{H}-5,33-34$ & 61.44 & 33.19 & 8.14 \\
\hline $7 \mathrm{H}-5,83-84$ & 61.94 & 44.39 & 6.58 \\
\hline
\end{tabular}

Table 1 (continued).

\begin{tabular}{|c|c|c|c|}
\hline $\begin{array}{l}\text { Core, section, } \\
\text { interval }(\mathrm{cm})\end{array}$ & $\begin{array}{l}\text { Depth } \\
\text { (mbsf) }\end{array}$ & $\begin{array}{l}\text { Calcium } \\
\text { carbonate } \\
(\%)\end{array}$ & $\begin{array}{c}\text { Si-opal } \\
(\%)\end{array}$ \\
\hline \multicolumn{4}{|l|}{ 111-677B- (Cont.) } \\
\hline $7 \mathrm{H}-5,133-134$ & 62.44 & 25.26 & 8.92 \\
\hline $7 \mathrm{H}-6,33-34$ & 62.94 & 23.62 & 8.11 \\
\hline $7 \mathrm{H}-6,83-84$ & 63.44 & 40.77 & 6.01 \\
\hline $7 \mathrm{H}-6,133-134$ & 63.94 & 55.26 & 5.74 \\
\hline $7 \mathrm{H}-7,33-34$ & 64.44 & 41.31 & 7.07 \\
\hline $8 \mathrm{H}-1,33-34$ & 64.94 & 23.62 & 10.24 \\
\hline $8 \mathrm{H}-1,83-84$ & 65.44 & 11.64 & 9.71 \\
\hline $8 \mathrm{H}-1,133-134$ & 65.94 & 15.43 & 9.89 \\
\hline $8 \mathrm{H}-2,33-34$ & 66.44 & 11.12 & 11.85 \\
\hline $8 \mathrm{H}-2,83-84$ & 66.94 & 13.48 & 10.09 \\
\hline $8 \mathrm{H}-2,133-134$ & 67.44 & 9.97 & 13.00 \\
\hline $8 \mathrm{H}-3,33-34$ & 67.94 & 26.47 & 8.60 \\
\hline $8 \mathrm{H}-3,83-84$ & 68.44 & 29.15 & 9.29 \\
\hline $8 \mathrm{H}-3,133-134$ & 68.94 & 19.98 & 9.47 \\
\hline $8 \mathrm{H}-4,31-32$ & 69.42 & 16.65 & 9.34 \\
\hline $8 \mathrm{H}-4,83-84$ & 69.94 & 20.44 & 10.18 \\
\hline $8 \mathrm{H}-4,133-134$ & 70.44 & 13.31 & 10.33 \\
\hline $8 \mathrm{H}-5,33-34$ & 70.94 & 14.46 & 11.26 \\
\hline $8 \mathrm{H}-5,83-84$ & 71.44 & 9.19 & 13.77 \\
\hline $8 \mathrm{H}-5,133-134$ & 71.94 & 12.10 & 12.20 \\
\hline $8 \mathrm{H}-6,33-34$ & 72.44 & 21.87 & 12.60 \\
\hline $8 \mathrm{H}-6,83-84$ & 72.94 & 14.32 & 12.24 \\
\hline $9 \mathrm{H}-1,33-34$ & 74.44 & 17.69 & 11.55 \\
\hline $9 \mathrm{H}-1,83-84$ & 74.94 & 27.69 & 9.43 \\
\hline $9 \mathrm{H}-1,133-134$ & 75.44 & 27.06 & 11.10 \\
\hline $9 \mathrm{H}-2,33-34$ & 75.94 & 10.30 & 12.37 \\
\hline $9 \mathrm{H}-2,83-84$ & 76.44 & 19.65 & 11.26 \\
\hline $9 \mathrm{H}-2,133-134$ & 76.94 & 22.36 & 12.93 \\
\hline $9 \mathrm{H}-3,33-34$ & 77.44 & 25.49 & 10.68 \\
\hline $9 \mathrm{H}-3,83-84$ & 77.94 & 25.74 & 12.13 \\
\hline $9 \mathrm{H}-3,133-134$ & 78.44 & 31.02 & 11.20 \\
\hline $9 \mathrm{H}-4,83-84$ & 79.44 & 41.43 & 10.13 \\
\hline $9 \mathrm{H}-4,133-134$ & 79.94 & 37.95 & 10.24 \\
\hline $9 \mathrm{H}-5,33-34$ & 80.44 & 34.93 & 13.23 \\
\hline $9 \mathrm{H}-5,83-84$ & 80.94 & 29.60 & 13.20 \\
\hline $9 \mathrm{H}-5,133-134$ & 81.44 & 39.55 & 10.47 \\
\hline $9 \mathrm{H}-6,33-34$ & 81.94 & 33.78 & 12.70 \\
\hline $9 \mathrm{H}-6,83-84$ & 82.44 & 29.41 & 12.27 \\
\hline $9 \mathrm{H}-6,133-134$ & 82.94 & 25.12 & 11.10 \\
\hline $10 \mathrm{H}-1,83-84$ & 84.44 & 12.33 & 12.65 \\
\hline $10 \mathrm{H}-1,133-134$ & 84.94 & 27.13 & 11.86 \\
\hline $10 \mathrm{H}-2,33-34$ & 85.44 & 31.98 & 8.65 \\
\hline $10 \mathrm{H}-2,83-84$ & 85.94 & 15.17 & 10.36 \\
\hline $10 \mathrm{H}-2,133-134$ & 86.44 & 15.59 & 10.10 \\
\hline $10 \mathrm{H}-3,33-34$ & 86.94 & 37.66 & 8.81 \\
\hline $10 \mathrm{H}-3,83-84$ & 87.44 & 56.92 & 5.13 \\
\hline $10 \mathrm{H}-3,133-134$ & 87.94 & 46.08 & 7.64 \\
\hline $10 \mathrm{H}-4,83-84$ & 88.94 & 46.31 & 7.24 \\
\hline $10 \mathrm{H}-4,133-134$ & 89.44 & 32.85 & 10.92 \\
\hline $10 \mathrm{H}-5,33-34$ & 89.94 & 32.50 & 13.98 \\
\hline $10 \mathrm{H}-5,83-84$ & 90.44 & 44.37 & 6.56 \\
\hline $10 \mathrm{H}-5,133-134$ & 90.94 & 48.76 & 6.95 \\
\hline $10 \mathrm{H}-6,33-34$ & 91.44 & 36.86 & 8.14 \\
\hline $10 \mathrm{H}-6,83-84$ & 91.94 & 48.52 & 6.59 \\
\hline $10 \mathrm{H}-6,133-134$ & 92.44 & 52.79 & 7.43 \\
\hline
\end{tabular}

\footnotetext{
a Samples are assigned sub-bottom depths according to the barrel sheets (Shipboard Scientific Party, 1983, 1988). The only exception from the depths indicated in the barrel sheets is in Core 111-677A-16X. It was obvious that the voids shown on the barrel sheets are not really portions of missing sediments (reported recovery was $190 \%$ ), but gaps that formed in the core liner during core retrieval.
}

coring process within each hole, (3) identify portions of the stratigraphic column that were sampled twice or unsampled within a hole, and (4) correct for sedimentation rate differences, fill in unsampled intervals with section from adjacent holes, and remove doubly sampled intervals. 
Table 2. Percent carbonate values used in this study of samples from DSDP Leg 69 Hole 504 in addition to "Karbonat-Bombe" determinations from the barrel sheets in Shipboard Scientific Party (1983).

\begin{tabular}{|c|c|c|}
\hline $\begin{array}{l}\text { Core, section, } \\
\text { interval }(\mathrm{cm})\end{array}$ & $\begin{array}{l}\text { Depth } \\
\text { (mbsf) }\end{array}$ & $\begin{array}{c}\text { Calcium } \\
\text { carbonate } \\
(\%)\end{array}$ \\
\hline $15-3,60-61$ & 75.21 & 16.52 \\
\hline $15-3,100-101$ & 75.61 & 24.35 \\
\hline $16-1,61-62$ & 76.62 & 36.37 \\
\hline $16-1,113-114$ & 77.14 & 11.08 \\
\hline $16-2,61-62$ & 78.12 & 37.17 \\
\hline $16-2,115-116$ & 78.66 & 22.22 \\
\hline $16-3,61-62$ & 79.62 & 32.35 \\
\hline $17-1,92-93$ & 81.33 & 34.40 \\
\hline $17-1,142-143$ & 81.83 & 40.72 \\
\hline $17-2,65-66$ & 82.56 & 25.85 \\
\hline $17-2,111-112$ & 83.02 & 42.36 \\
\hline $17-3,63-64$ & 84.04 & 19.02 \\
\hline $18-1,64-65$ & 85.45 & 21.93 \\
\hline $18-1,111-112$ & 85.92 & 12.49 \\
\hline $18-2,61-62$ & 86.92 & 40.69 \\
\hline $18-2,115-116$ & 87.46 & 55.89 \\
\hline $18-3,67-68$ & 88.48 & 52.06 \\
\hline $19-1,68-69$ & 89.89 & 27.37 \\
\hline $19-1,117-118$ & 90.38 & 36.50 \\
\hline $19-2,66-67$ & 91.37 & 42.33 \\
\hline $19-2,111-112$ & 91.82 & 48.20 \\
\hline $19-3,11-12$ & 92.32 & 38.20 \\
\hline $20-1,67-68$ & 94.28 & 44.80 \\
\hline $20-1,117-118$ & 94.78 & 37.55 \\
\hline $20-2,66-67$ & 95.77 & 49.05 \\
\hline $20-2,114-115$ & 96.25 & 42.61 \\
\hline $20-3,68-69$ & 97.29 & 51.76 \\
\hline $20-3,117-118$ & 97.78 & 42.26 \\
\hline $21-1,67-68$ & 98.68 & 60.86 \\
\hline $21-1,117-118$ & 99.18 & 68.19 \\
\hline $21-2,67-68$ & 100.18 & 34.93 \\
\hline $21-2,118-119$ & 100.69 & 34.39 \\
\hline $21-3,67-68$ & 101.68 & 63.26 \\
\hline $21-3,114-115$ & 102.15 & 42.01 \\
\hline $25-1,57-58$ & 116.18 & 52.93 \\
\hline $25-2,8-9$ & 117.19 & 46.57 \\
\hline $25-2,107-108$ & 118.18 & 41.63 \\
\hline $26-1,37-38$ & 120.38 & 53.31 \\
\hline $26-2,6-7$ & 121.57 & 54.77 \\
\hline $26-2,108-109$ & 122.59 & 49.04 \\
\hline $27-1,37-38$ & 124.78 & 46.78 \\
\hline $27-2,7-8$ & 125.98 & 51.35 \\
\hline $28-1,60-61$ & 129.41 & 42.44 \\
\hline $28-1,117-118$ & 129.98 & 42.34 \\
\hline $28-2,67-68$ & 130.98 & 49.01 \\
\hline $29-1,62-63$ & 133.83 & 51.18 \\
\hline $29-1,110-111$ & 134.31 & 46.03 \\
\hline $29-2,85-86$ & 135.56 & 50.08 \\
\hline $29-3,67-68$ & 136.88 & 47.17 \\
\hline $29-3,118-119$ & 137.39 & 48.80 \\
\hline $30-1,67-68$ & 138.28 & 45.21 \\
\hline $30-1,117-118$ & 138.78 & 53.34 \\
\hline $30-2,61-62$ & 139.72 & 53.17 \\
\hline $30-2,114-115$ & 140.25 & 37.17 \\
\hline $30-3,54-55$ & 141.15 & 48.65 \\
\hline $31-1,37-38$ & 142.38 & 59.44 \\
\hline $31-2,79-80$ & 144.30 & 49.66 \\
\hline $32-1,6-7$ & 146.47 & 66.30 \\
\hline $32-1,61-62$ & 147.02 & 60.05 \\
\hline $32-2,67-68$ & 148.58 & 61.25 \\
\hline $32-2,115-116$ & 149.06 & 68.03 \\
\hline $33-1,60-61$ & 151.41 & 60.63 \\
\hline $33-1,116-117$ & 151.97 & 71.09 \\
\hline $33-2,30-31$ & 152.61 & 67.63 \\
\hline $34-1,76-77$ & 155.97 & 22.74 \\
\hline $34-2,65-66$ & 157.36 & 58.13 \\
\hline $34-2,113-114$ & 157.84 & 59.29 \\
\hline $35-1,63-64$ & 160.24 & 48.70 \\
\hline
\end{tabular}

Table 2 (continued).

\begin{tabular}{lcc}
\hline $\begin{array}{l}\text { Core, section, } \\
\text { interval }(\mathrm{cm})\end{array}$ & $\begin{array}{c}\text { Depth } \\
\text { (mbsf) }\end{array}$ & $\begin{array}{c}\text { Calcium } \\
\text { carbonate } \\
(\%)\end{array}$ \\
\hline $35-1,113-114$ & 160.74 & 38.74 \\
$35-2,62-63$ & 161.73 & 49.84 \\
$35-2,112-113$ & 162.23 & 61.74 \\
$35-3,60-61$ & 163.21 & 63.36 \\
$36-1,60-61$ & 164.61 & 74.01 \\
$36-1,110-111$ & 165.11 & 71.39 \\
$36-2,69-70$ & 166.20 & 66.08 \\
$36-2,115-116$ & 166.66 & 73.73 \\
$37-1,65-66$ & 168.06 & 74.39 \\
$37-1,115-116$ & 169.56 & 66.61 \\
$37-2,60-61$ & 170.51 & 69.81 \\
$37-2,111-112$ & 171.02 & 70.57 \\
$38-1,64-65$ & 173.45 & 72.62 \\
$38-1,111-112$ & 173.92 & 70.27 \\
$38-2,63-64$ & 174.94 & 74.62 \\
$38-2,114-115$ & 175.45 & 67.16 \\
$38-3,60-61$ & 176.41 & 73.95 \\
$40-1,60-61$ & 182.21 & 76.68 \\
$40-1,116-117$ & 182.77 & 74.08 \\
$40-2,66-67$ & 183.77 & 67.15 \\
$41-1,76-77$ & 186.77 & 83.63 \\
$41-1,123-124$ & 187.24 & 60.75 \\
$41-2,61-62$ & 188.12 & 91.88 \\
$41-3,68-69$ & 188.99 & 75.49 \\
$41-3,114-115$ & 189.45 & 82.50 \\
$42-1,8-9$ & 190.09 & 76.00 \\
$42-1,63-64$ & 190.64 & 84.41 \\
$42-1,116-117$ & 191.17 & 64.63 \\
$42-2,65-66$ & 192.16 & 77.93 \\
$43-1,61-62$ & 194.62 & 74.53 \\
$43-1,115-116$ & 195.16 & 76.29 \\
\hline & & \\
\hline
\end{tabular}

\section{Correlation by Color}

Color changes within cores can be used to match stratigraphic sections in deep-sea cores (e.g., Ruddiman et al., 1986). This method works well when there are distinct, rather than gradual or continuous, color changes associated with lithologic differences that are easily recognized in core photos. The number of color changes observed in Holes 677A and 677B (documented on shipboard lithologic core description forms) is shown in Figure 1. It is clear that a correlation based on color changes cannot be achieved because similar strata are not easily recognized. In addition, the mottled and bioturbated appearance of these cores makes it difficult to visually recognize distinct strata with the exception of layers of volcanic ash. Therefore, other data must be used to make precise correlations.

\section{Graphic Correlation}

The biostratigraphy and lithostratigraphy (ash layers) of Sites 504 and 677 were used for first-order estimates of the completeness of the holes. Shaw plots (Shaw, 1964) were constructed (Fig. 2) using biostratigraphic data (Table 3). Each datum level was resolved to $50 \mathrm{~cm}$ and is considered reliable (see Alexandrovich, this volume). Datum levels are assumed to be isochronous within the sampling interval because diachroneity is implausible between such closely spaced holes. The Shaw plots show significant departure from one-to-one correlation between holes: the line of correlation can not be represented by a straight line with a slope of one, which would be expected with complete, identical sections.

\section{Calcium Carbonate Stratigraphy}

Downhole changes in the concentration of calcium carbonate have been used to identify time-synchronous horizons in sev- 


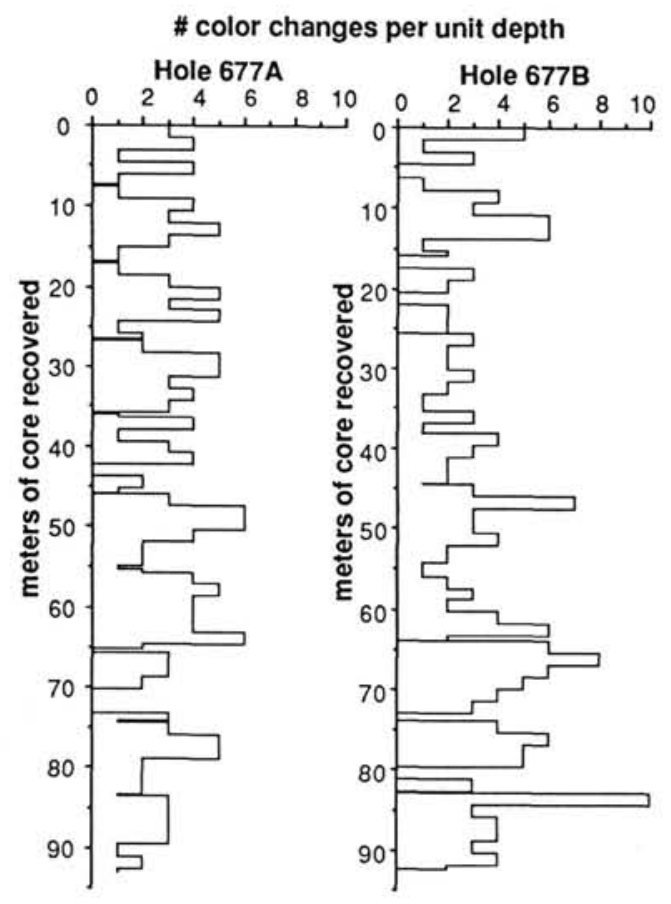

Figure 1. Histogram of the number of color changes observed per core section of Holes 677A and 677B. Units are of meters of core recovered, not recorded sub-bottom depth.

Table 3. Datum levels used for stratigraphic correlation of DSDP Leg 69 Hole 504 and ODP Leg 111 Holes 677A and 677B.

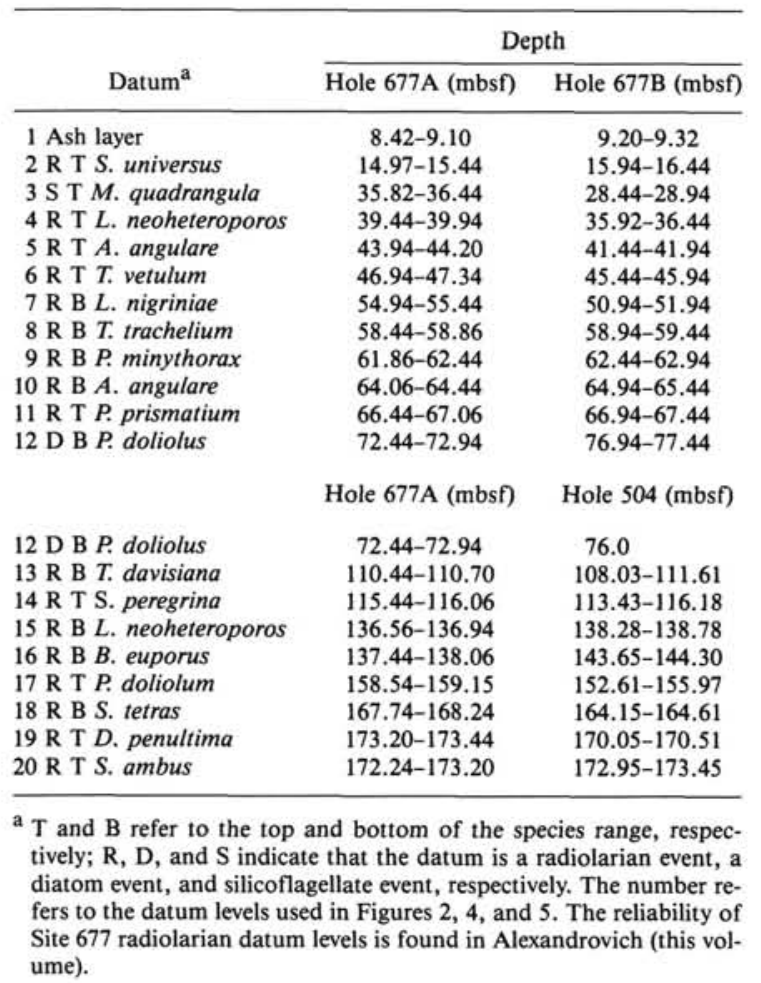

eral equatorial Pacific holes (e.g., Hays et al., 1969; Saito et al., 1975; Dunn and Moore, 1981; Pisias et al., 1985). Fluctuations in carbonate content can occur on glacial to interglacial time scales and are caused by variations in carbonate saturation and
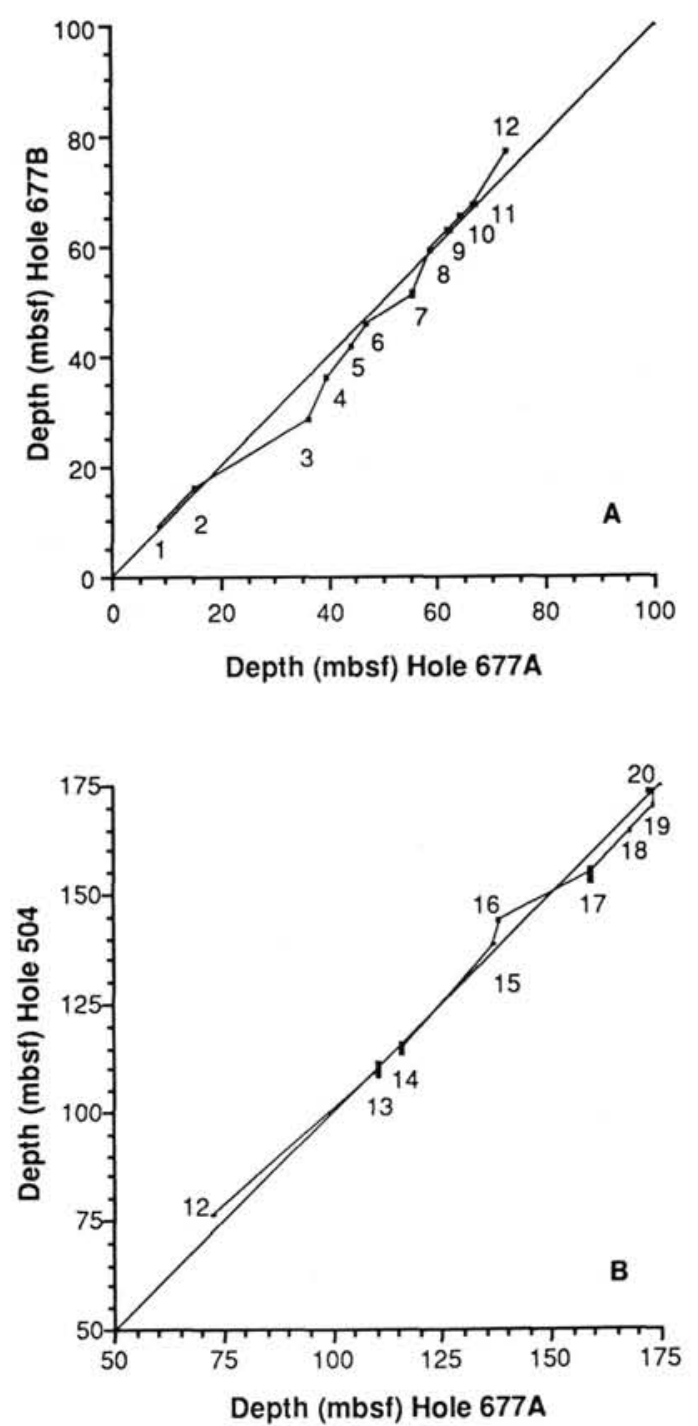

Figure 2. Shaw plot showing stratigraphic correlation between Holes 677A and 677B (A) and between Holes 677A and 504 (B). See Table 3 for identification of datum levels. Straight lines indicate perfect one-toone correlations.

productivity in the water column at the time of deposition. Carbonate variations in Holes 504, 677A, and 677B were used to correlate equivalent horizons. In Holes 677A and 677B percent records of $\mathrm{Si}$-opal and the nonbiogenic component were correlated and used as independent checks on the correlations made using the carbonate data. Given the equivalent lithologies of these holes, correlations were based on absolute values of percent carbonate, percent $\mathrm{Si}$-opal, and percent nonbiogenic, as well as the patterns of peaks and troughs.

\section{Inverse Correlation Technique-CORPAC}

If perfect recovery were obtained, the deviations observed from a perfect line of correlation between Holes 677A and 677B could be caused by subtle differences in sedimentation rates caused by topography (ponding of sediments) or diagenetic overprints that might be an effect of heat flow through the sediments causing differential compaction. Using continuous stratigraphic records, such as percent carbonate and percent $\mathrm{Si}$-opal, it is possible to apply methods of signal correlation to identify where sedimentation rate differences exist and to correct for them. CORPAC, a signal correlation program that uses the in- 
verse method of correlation (Martinson et al., 1982), was used to examine in detail the line of correlation (mapping function) between Holes 677A and 677B and between Holes 677A and 504.

Briefly, CORPAC works as follows: it assumes that the two time series to be correlated are similar except that one has undergone distortion (stretching and/or squeezing) of the depth or time axis relative to the other. The relationship between the signals can be described by a "mapping" function that relates the corresponding portions of the independent variable axes of the two signals. The shape of the mapping function can be parameterized as a linear trend modified by a Fourier sine series. The technique iteratively determines those Fourier coefficient values that produce a mapping function with a shape that maximizes the correlation coefficient between the two time series.

The records of weight percent carbonate, Si-opal, and nonbiogenic from Hole 677B and the top $91.7 \mathrm{~m}$ from Hole 677A were used as input for CORPAC. Because these curves display different characters and are independent of one another (nonbiogenic is an exception) they provide checks on the resultant correlations because there can be only one correct stratigraphic correlation. Several experiments were performed to examine the data. These tests included autocorrelation using no tie-lines, autocorrelation using endpoints as ties, autocorrelation using single tie-lines based on biostratigraphic datum levels, and autocorrelation using sets of tie-lines between selected peaks and troughs identified by their proximity to stratigraphic datum levels. Distinctive character changes recognized in the records not near biostratigraphic levels were also used in the tie-line sets. The mapping functions obtained from these correlations are remarkably similar in that major slope changes occur in the same direction and at the same stratigraphic level in the various runs. These levels coincide with core breaks (Fig. 3). This suggests that signal differences were caused by coring complications resulting in missing or doubly sampled intervals at core breaks.

\section{Visual Matching}

Because it is known that sediments can be lost or double cored at core breaks (Ocean Drilling Program, 1987) and COR-

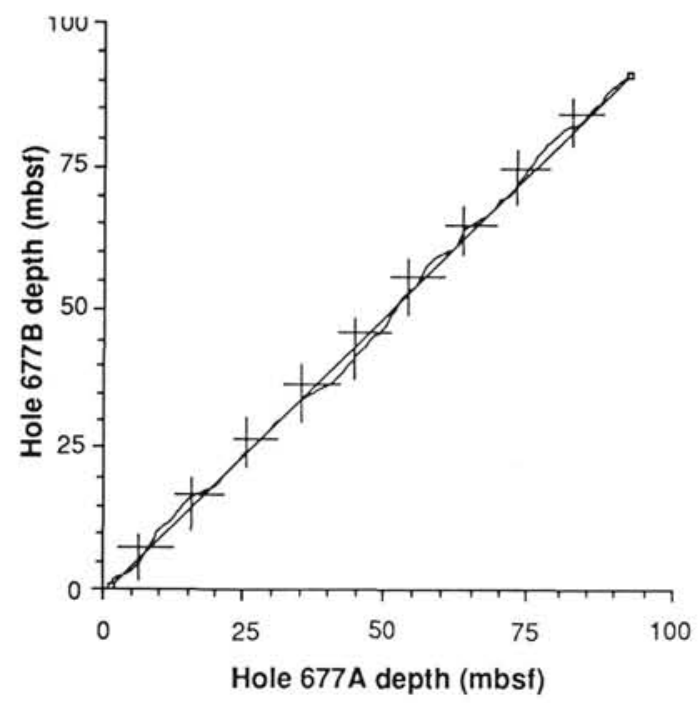

Figure 3. Mapping function produced by correlation of calcium carbonate records of Holes $677 \mathrm{~A}$ and $677 \mathrm{~B}$ using a full set of tie-lines and 50 coefficients. Coherence is 0.86 . Vertical and horizontal lines indicate depths of core breaks in Holes 677A and 677B, respectively.
PAC mapping functions show deviations at core break depths, we have taken as a first approximation the assumption that deviations of the stratigraphic datum levels from perfect correlation were caused by uncertainties in core retrieval rather than sedimentation rate differences. Due to their proximity, the three holes are thus assumed to have identical sedimentation rates and patterns. In support of this, no slumping or erosive features have been identified. Bioturbation is the only visible disturbance in the sediments, but this appears to be uniform and should not inflict major differences on the nature of the sediments between holes.

To determine the extent of missing and double-cored stratigraphic sections in Holes 504, 677A, and 677B, we studied the percent carbonate, Si-opal, and the nonbiogenic component records on a larger scale in order to examine the character of the records, particularly at core breaks. The records were plotted on an expanded scale of 1 in. $=2 \mathrm{~m}$, with biostratigraphic and ash layer datum levels noted in the correct stratigraphic position.

Each core was plotted separately (the lowest data point in a core was not connected by a line to the highest data point in an adjacent core) so that any overlap or missing sections at the core breaks could be identified by comparing the patterns of the Hole 677A and Hole 677B records. These records were overlaid on a light table and matched by eye using the biostratigraphic information (Table 3) as correlation control. The goal was to identify the missing or double-cored portions of the stratigraphic column at Site 677 , so no stretching or squeezing was performed on the records except at core breaks. Where it was unclear whether section should be added or subtracted at a core break, we either made no adjustment or added section rather than subtracting.

The carbonate record was patched together first. This record has the most structure and the smallest analytical uncertainty. A patching session consisted of matching patterns visually and deciding if, and how much of, the stratigraphic section was missing or duplicated. Because the cores in the two sites were staggered in depth by $2.5 \mathrm{~m}$, missing sections from core breaks in one hole could be identified in the other hole. However, the depth difference between breaks in the two holes did not remain constant with depth. After the carbonate records were patched together, the biogenic opal records and finally the percent nonbiogenic records were patched together. Care was taken not to bias the way in which the cores were patched. No two sessions immediately followed one another, and sessions were usually performed on different days. Nine solutions resulted from the efforts of the two investigators working with three data sets.

Figure 4 illustrates how the carbonate records from Holes $677 \mathrm{~A}$ and $677 \mathrm{~B}$ were compared and patched. The patterns and absolute values of the records from each core were matched using the datum level information as guides. For first-order correlation, these datum levels provided sufficient information to match age-equivalent cores, but in the final high-resolution correlation, several of the datum levels became offset stratigraphically between the two cores. The datum levels that show stratigraphic offset between the patched records $(4,7$, and 12 of Table 3) can be argued to be equivocal, due mainly to the low abundance of the species at this site (see Alexandrovich, this volume).

The visual patching sessions did not produce a unique solution. High confidence was placed on solutions that occurred more commonly using the different data sets. The amount of stratigraphic section that was added or subtracted at core breaks in each of the nine attempts at visual correlation was measured and averaged (Table 4). The patched data account for $3.1 \%$ and $4.7 \%$ of unsampled material in Holes $677 \mathrm{~A}$ and $677 \mathrm{~B}$, respectively. The new patched records were recorrelated using CORPAC and resulted in an increase in correlation coefficient from 0.86 to 0.87 . An example of the tie-line sets used for autocorre- 

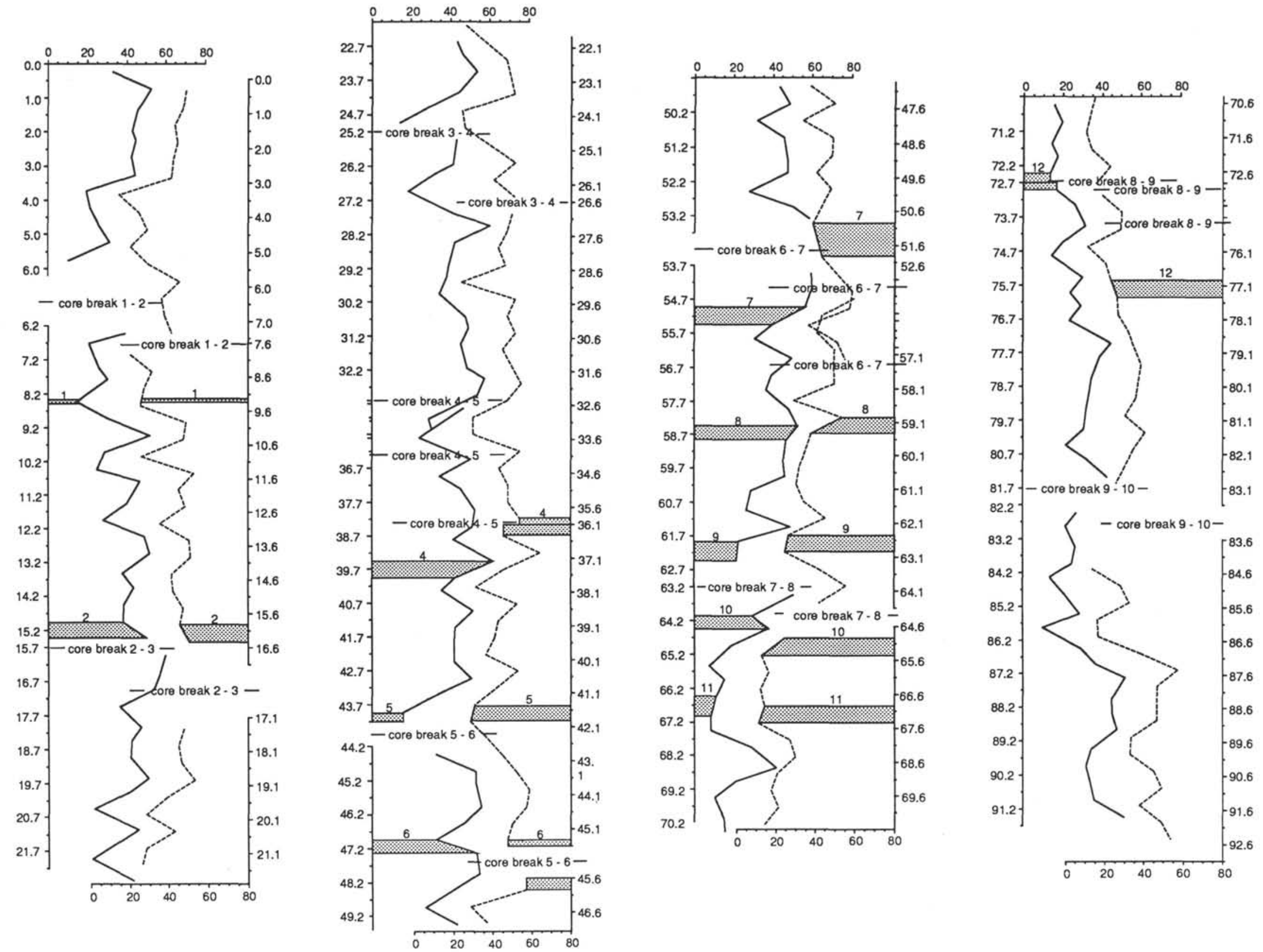

Figure 4. Example of visual patching procedure on weight percent calcium carbonate records of Holes 677A (solid line) and 677B (dashed line). Depth is in meters below seafloor. Shaded stratigraphic information is numbered as in Table 3. Missing section occurs where there is a break in the depth scale at core breaks, and doubly cored intervals occur where the depth scale overlaps (core breaks are indicated twice). 
Table 4. Adjustments made to depth at core breaks after visual patching of Holes 677A and 677B.

\begin{tabular}{ccc}
\hline Core break & Adjustment to Hole 677A $(\mathrm{m})$ & Adjustment to Hole 677B $(\mathrm{m})$ \\
\hline $0 / 1$ & No change & +0.50 \\
$1 / 2$ & +1.25 & No change \\
$2 / 3$ & No change & +1.50 \\
$3 / 4$ & No change & No change \\
$4 / 5$ & -1.65 & No change \\
$5 / 6$ & +0.70 & +0.90 \\
$6 / 7$ & +0.90 & -2.30 \\
$7 / 8$ & No change & +0.50 \\
$8 / 9$ & No change & -1.20 \\
$9 / 10$ & No change & +1.00
\end{tabular}

Total adjustment over the 10-core stratigraphic section

$$
+1.20
$$$$
+0.90
$$

Note: + indicates section added to and - indicates section removed from the stratigraphic column.

lation is shown in Figure 5 on the opal record and does not include datum levels that do not occur at the same stratigraphic level after patching. The resulting mapping function is almost perfectly flat (Fig. 6), adding higher confidence to the visual stratigraphic adjustments. The final correlation of the records is shown in Figure 7.

The Pliocene portion of Hole 504 (62.8-177.2 mbsf) was correlated to Hole 677A (63.2-182.8 mbsf) using carbonate data and one diatom and eight radiolarian datum levels as guides (Table 3). Hole $677 \mathrm{~A}$ was chosen as the reference section because there are approximately two core breaks in Hole 504 to every one core break in Hole 677A, and Hole 504 sediments are more disturbed than Hole 677A sediments, which resulted in a much more incomplete record. The resulting mapping function (Fig. 8) shows large deviations from a perfect correlation. These records were then patched visually and adjusted (Table 5). No adjustments were made to Hole 677A. After patching, correlation between Holes 504 and $677 \mathrm{~A}$ is improved significantly (Fig. 9), and the correlation coefficient increases from 0.90 to 0.93 and yields a nearly flat mapping function (Fig. 10). The patched-in data amount to $14.16 \%$ of the unsampled material.

\section{DISCUSSION}

Quantitative records of sedimentary properties combined with high-resolution biostratigraphic information provide excellent correlation tools. The carbonate and $\mathrm{Si}$-opal records of Holes $504,677 \mathrm{~A}$, and $677 \mathrm{~B}$ contain enough information to determine where stratigraphic sections are incomplete. This study shows
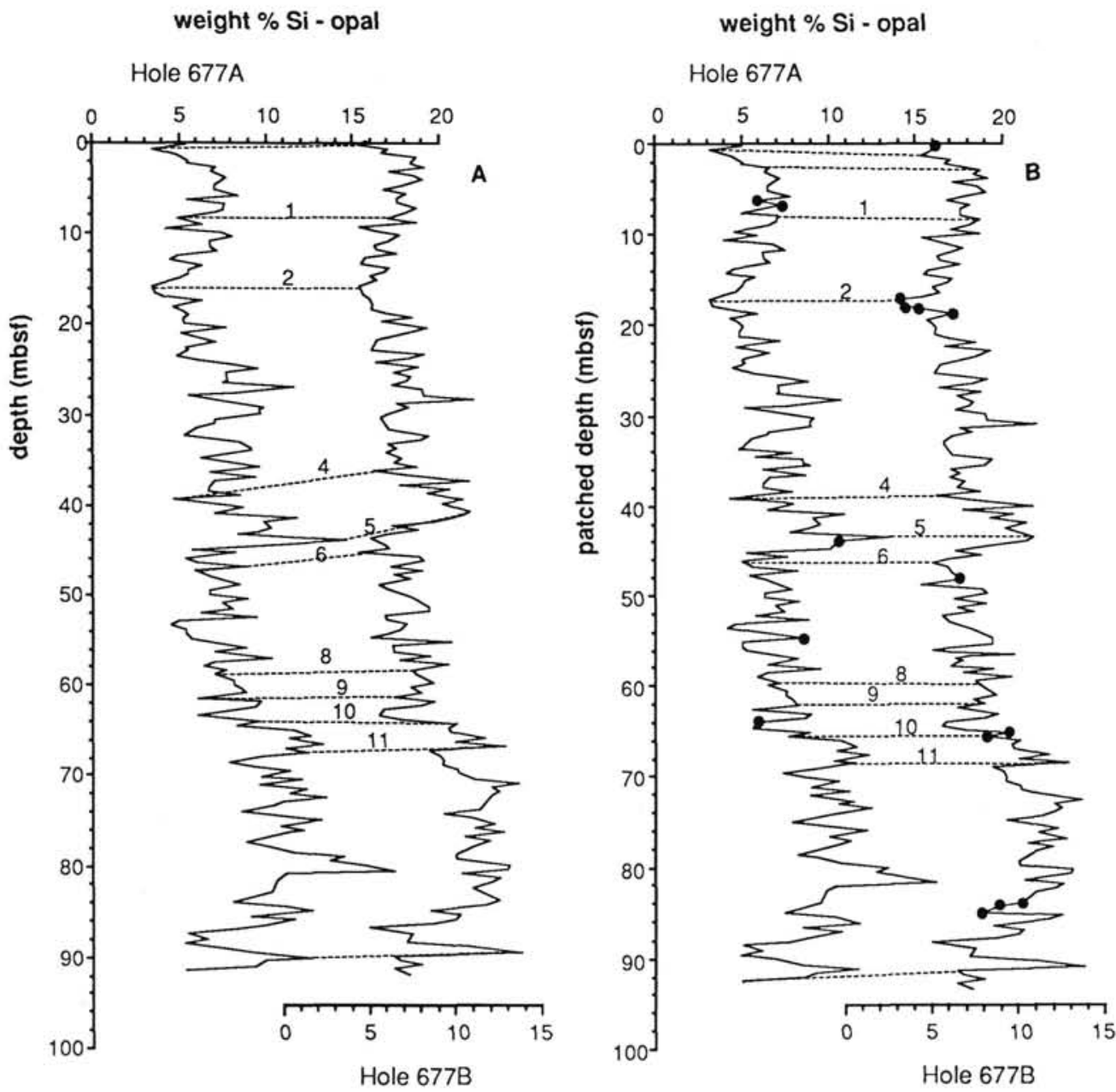

Figure 5. Tie-lines on percent Si-opal records from Holes 677A and 677B (on the left and right, respectively, of each plot) used for nonlinear correlation of nonpatched records $(\mathbf{A})$ and patched records $(\mathbf{B})$. Numbered tielines indicate the stratigraphic datum level in Table 3. Unlabeled tie-lines are determined by distinct lithologic features. Patched-in values are indicated with dots. 


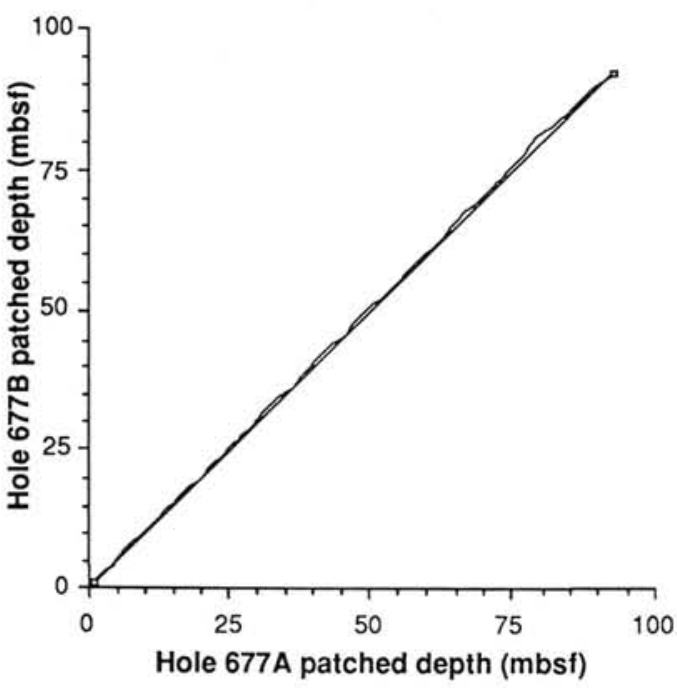

Figure 6. Mapping function produced by correlation of patched calcium carbonate records of Holes 677A and 677B using a full set of tie-lines and 29 coefficients. Coherence is 0.87 . that significant portions of stratigraphic sections can be both lost and double cored at core breaks during hydraulic piston coring even in calm seas. During coring on Leg 111 the sea state was relatively calm, with 3 - to 6 -ft seas, 5 - to 9 -ft swells, and a ship heave of $2 \mathrm{ft}$.

There are several reasons for missing or double-coring section during hydraulic piston coring. Section can be lost at core breaks by advancing the drill string too far before firing the corer. This results in losing sediments from the top of the section being cored. Section may also be lost as a result of ship heave during piston firing. If the ship heaves downward before firing, section may be lost from the top of the core, but if the ship heaves upward, section may be lost from the bottom of the core. The length of the intervals lost and double cored in Holes $677 \mathrm{~A}$ and $677 \mathrm{~B}$ ranges from less than $1 \mathrm{~m}$ to slightly over $2 \mathrm{~m}$. These intervals are comparable to the ship's heave and may be a result of it. Double coring is more difficult to explain when, as in the case of Site 677 sediments, the sediments show little evidence of coring disturbance. When pipe joint length added to the drill string is less than the penetration of the last core (ODP policy is to advance the drill string the length of the pipe joint), this may result in sampling slump at the top of the core, but the doubly cored material by nature should show obvious drilling disturbance. Doubly sampled section may also occur if the sediments that line the hole move in laterally to fill the vacancies left original signals

$0 \quad 1020 \quad 30 \quad 40 \quad 5060 \quad 70$

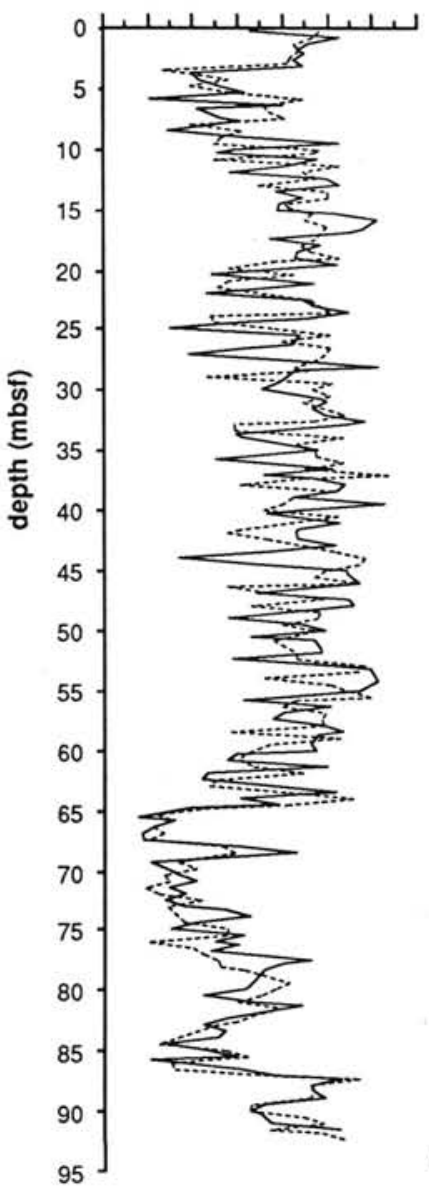

correlated signals

$\begin{array}{llllllll}0 & 10 & 20 & 30 & 40 & 50 & 60 & 70\end{array}$

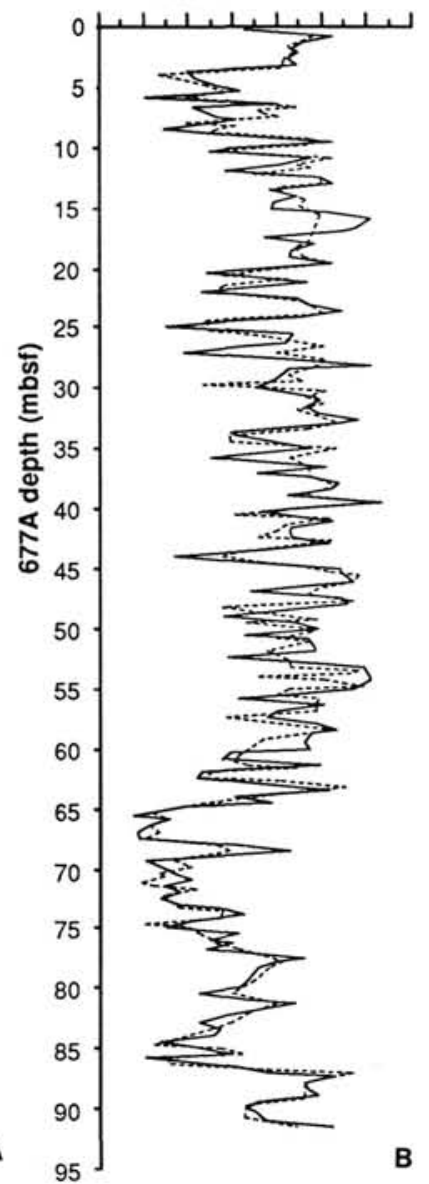

patched signals

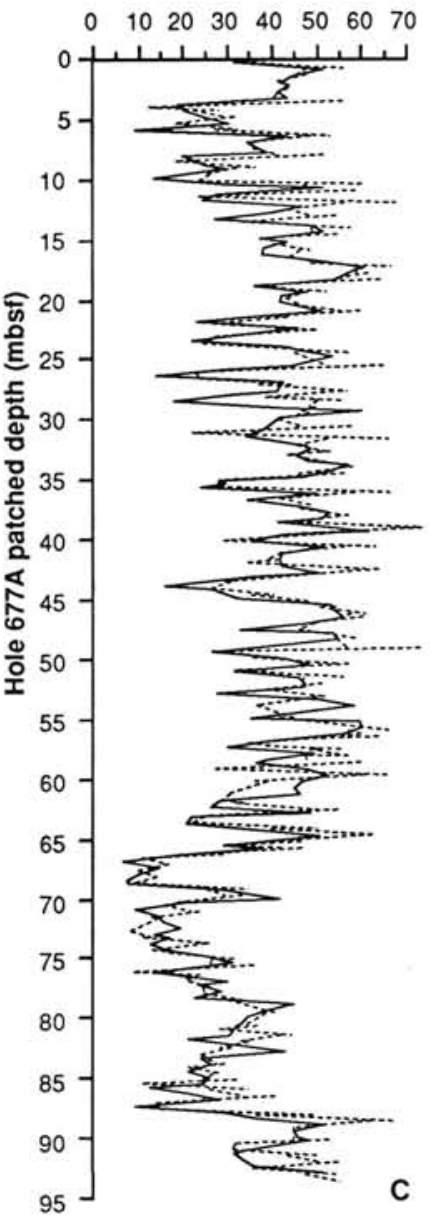

patched and correlated

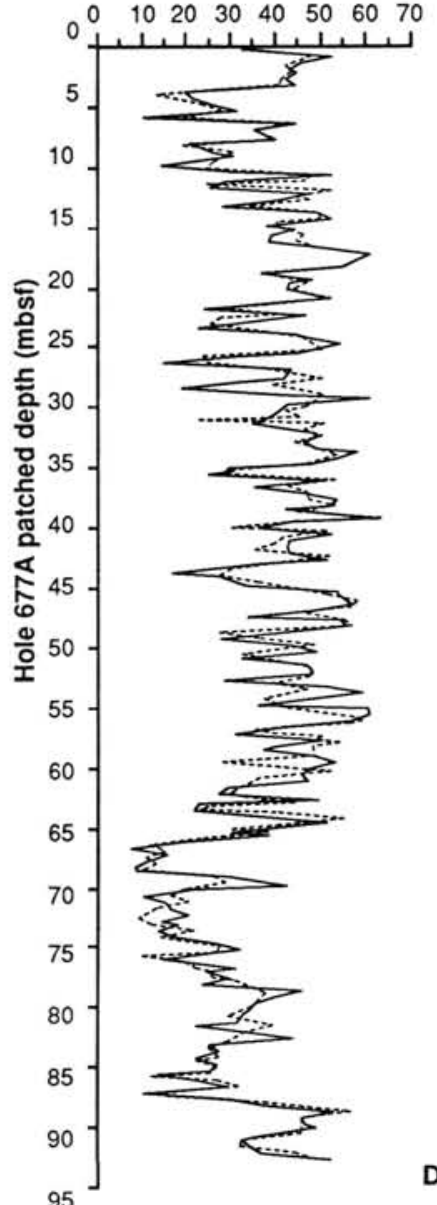

Figure 7. Percent calcium carbonate of Holes 677A (solid line) and 677B (dashed line). A. Original signals plotted against sub-bottom depth. B. Correlated signals plotted against sub-bottom depth in Hole 677A. C. Patched signals. Sub-bottom depths were adjusted as indicated in Table 4. D. Patched and correlated signals plotted against Hole 677A adjusted sub-bottom depth. 


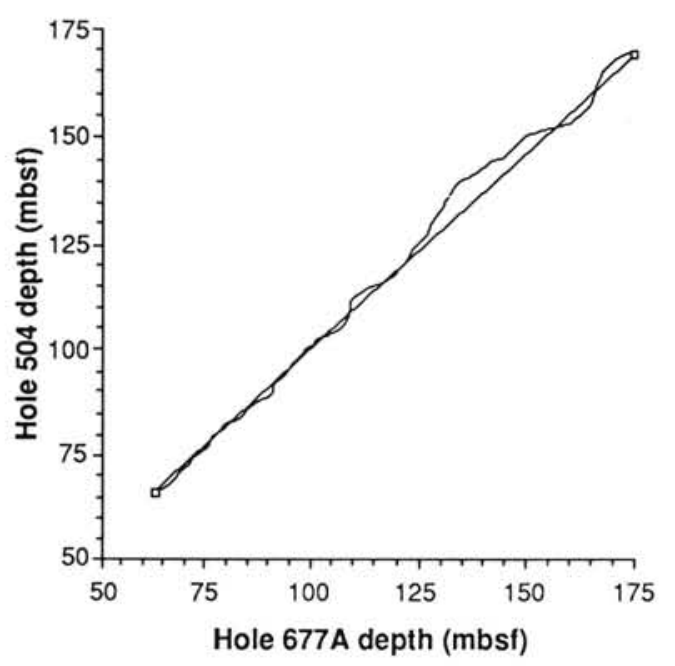

Figure 8. Mapping function produced by correlation of nonpatched calcium carbonate records of Holes 677A and 504 using a full set of tielines and 50 coefficients. Coherence is 0.90 . Straight line indicates oneto-one correlation.

\begin{abstract}
Table 5. Adjustments made to depths at core breaks in Hole 504 after visual patching of Holes 504 and $677 \mathrm{~A}$.
\end{abstract}

\begin{tabular}{cc}
\hline Core break & Adjustment to Hole $504(\mathrm{~m})$ \\
\hline $13 / 14$ & No change \\
$14 / 15$ & -1.50 \\
$15 / 16$ & +1.50 \\
$16 / 17$ & +0.25 \\
$17 / 18$ & +1.50 \\
$18 / 19$ & No change \\
$19 / 20$ & -1.80 \\
$20 / 21$ & +1.10 \\
$21 / 22$ & +1.70 \\
$22 / 23$ & +0.50 \\
$23 / 24$ & +0.40 \\
$24 / 25$ & -2.75 \\
$25 / 26$ & -1.00 \\
$26 / 27$ & No change \\
$27 / 28$ & +0.40 \\
$28 / 29$ & -2.50 \\
$29 / 30$ & -0.70 \\
$30 / 31$ & +3.15 \\
$31 / 32$ & +2.60 \\
$32 / 33$ & +1.20 \\
$33 / 34$ & +1.90 \\
$34 / 35$ & No change \\
$35 / 36$ & No change \\
$36 / 37$ & -1.50 \\
$37 / 38$ & -1.20 \\
Total adjustment over the 25 -core \\
stratigraphic section \\
& \\
& +3.25 \\
Note: + indicates section added to and - \\
graphic column. & \\
& \\
& \\
& \\
&
\end{tabular}

by coring. This may happen in shallow portions of the hole where sediments have high water contents or in deeper portions of the hole where overburden pressures may be high. We have no firm evidence that this occurs, but it should be tested by recoring an interval already cored. It may also be possible for the drill string to shift laterally within the sediments. If the ship heaves upward before the drill string is advanced, and the pipe shifts laterally, the top section of the next core would duplicate the bottom section of the previous core. Lateral shifting of the drill string may occur in soft shallow sediments, but as the drill string penetrates deeper into the sediments lateral shifting would seem more unlikely.

As a result of missing or double-coring stratigraphic section during piston coring, the relative depth of the staggered core breaks between double-cored holes does not remain constant. For instance, the patched sub-bottom depth between the first two cores in Hole $677 \mathrm{~A}$ is $6.2 \mathrm{~m}$ and $8.6 \mathrm{~m}$ in Hole $677 \mathrm{~B}$, a $2.4 \mathrm{~m}$ difference, whereas the patched sub-bottom depth between Cores $9 \mathrm{H}$ and $10 \mathrm{H}$ in Hole $677 \mathrm{~A}$ is $83.4 \mathrm{~m}$ and $83.5 \mathrm{~m}$ in Hole $677 \mathrm{~B}$, a difference of only $10 \mathrm{~cm}$, which is essentially zero offset within the sampling interval. Zero offset makes it impossible to locate and fill in missing stratigraphic section. For this reason triple coring is recommended. We also strongly recommend that the length of each drill pipe joint added be accurately measured, so there would be no chance of advancing the drill string beyond the amount of maximum piston penetration. As an alternative to triple coring, and because ship heave is a strong determinant in coring recovery, the drill string could be advanced less the amount of ship heave in order to ensure that no portion of a stratigraphic section is missed during coring. For example, if the ship was heaving $2 \mathrm{~m}$, the drill string should be advanced $7.8 \mathrm{~m}$ while the piston fires its full 9.5-m stroke. This would result in cores with slump at the top; however, this slump would be an indicator of complete stratigraphic recovery.

\section{SUMMARY}

First-order correlations between Holes 504, 677A, and 677B were made using the traditional Shaw method. Initial correlations (tie points) were determined using biostratigraphic control. Shaw plots do not show one-to-one depth relationships that are expected of holes that are in close proximity to one another. Apparent deviations from the line of correlation are due to coring complications rather than differences in the sedimentary records.

Detailed records of percent carbonate from DSDP Hole 504 and ODP Holes 677A and 677B and percent Si-opal from Holes $677 \mathrm{~A}$ and $677 \mathrm{~B}$ are used as correlation signals. Using this data in CORPAC, the correlation obtained produces a better matching of the records than when plotted by original sub-bottom depths. This correlation is not adequate, however, because CORPAC cannot correct a signal where portions of the stratigraphic column are missing. Correlations determined without consideration of missing or double-cored sections result in erroneously equating portions of the stratigraphic column that are not synchronous. For this reason, the records of percent carbonate, Si-opal, and the nonbiogenic component were patched together visually to create more complete records, using biostratigraphic datum levels, ash layers, absolute values, and the structure of the records as guides. Deviations from one-to-one correlation in CORPAC mapping functions verified the necessity for adjustments at each core break. The patched records were recorrelated using CORPAC producing a flat mapping function.

The results of this study indicate that a significant section of sediment may be lost or double cored at core breaks during hydraulic piston coring (up to $2 \mathrm{~m}$ in this study). This results in a significant alteration of the stratigraphic record. When complete stratigraphic sections are desired, concentrated effort must be made to ensure the retrieval of double- or preferably triplecored holes, good paleomagnetic and physical-property data, high-resolution biostratigraphy, and carefully planned and documented coring procedures. At high latitudes, paleomagnetic information can supplement biostratigraphy. Where color changes are subtle and cannot be recognized by eye, geochemical properties such as percent calcium carbonate must be used for correla- 

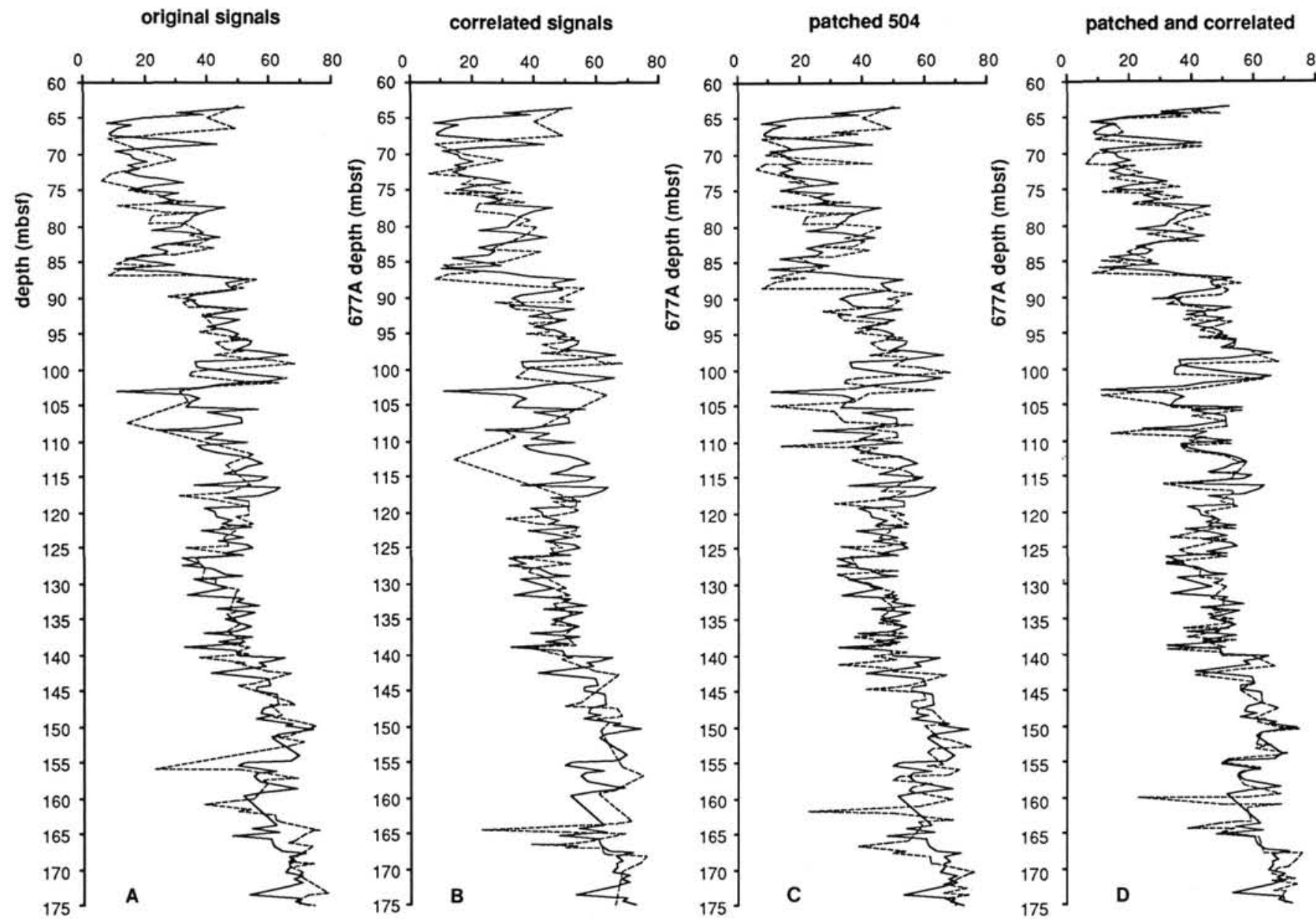

Figure 9. Percent calcium carbonate of Holes 677A (solid line) and 504 (dashed line). A. Original signals plotted against sub-bottom depth. B. Correlated signals plotted against sub-bottom depth in Hole 677A. C. Original Hole 677A and patched Hole 504 signals plotted against sub-bottom depth in Hole 677A. Sub-bottom depths of Hole 504 were adjusted as indicated in Table 6. D. Patched and correlated signals plotted against Hole 677A subbottom depth.

tion and should be determined aboard ship in order to determine stratigraphic continuity in real time. This will aid in procedural decisions about coring. When it is not possible to gather geochemical data, high-resolution magnetic susceptibility measurements may be used as a substitute and can be retrieved quickly, at small sample intervals, which will leave cores intact for future scientific investigations.

\section{ACKNOWLEDGMENTS}

The authors thank L. Burckle for the diatom and silicoflagellate datum levels, and C. Sancetta, P. Froelich, and L. Burckle for reviews of the manuscript. This work was funded by USSAC proposal TAMRF P.O. \#20055 and NSF Grant 0CE86-00744. This is Lamont-Doherty Geological Observatory contribution no. 4525 .

\section{REFERENCES}

Dunn, D. A., and Moore, T. C., Jr., 1981. Late Miocene-Pliocene (magnetic Epoch 9-Gilbert magnetic Epoch) calcium-carbonate stratigraphy of the equatorial Pacific Ocean. Geol. Soc. Am. Bull., 92:408451.

Hays, J. D., Saito, T., Opdyke, N. D., and Burckle, L. H., 1969. Pliocene-Pleistocene sediments of the equatorial Pacific: their paleomagnetic, biostratigraphic, and climatic record. Geol. Soc. Am. Bull., 80:689-694.

Jones, G. A., and Kaiteris, P., 1983. A vacuum-gasometric technique for rapid and precise analysis of calcium carbonate in sediments and soils. J. Sediment. Petrol., 53:655-660.
Martinson, D. G., Menke, W., and Stoffa, P., 1982. An inverse approach to signal correlation. J. Geophys. Res., 87:4807-4818.

Mortlock, R. E., and Froelich, P. N., in press. A simple method for the rapid determination of biogenic opal in pelagic marine sediments. Deep-Sea Res., Part A.

Müller, G., and Gastner, M., 1971. The "Karbonat-Bombe," a simple device for the determination of the carbonate content in sediments, soils, and other materials. Neues Jahrb. Mineral. Monatsh., 10:466469.

Ocean Drilling Program, 1987. Shipboard Scientists Handbook: ODP Tech. Note, 3.

Pisias, N. G., Barron, J. A., Nigrini, C. A., and Dunn, D. A., 1985. Stratigraphic resolution of Leg 85 drill sites: an initial analysis. In Mayer, L., Theyer, F., Thomas, E., et al., Init. Repts. DSDP, 85: Washington (U.S. Govt. Printing Office), 675-708.

Raymo, M. E., Ruddiman, W. F., Backman J., Clement, B. M., and Martinson, D. G., 1989. Late Pliocene variation in Northern Hemisphere ice sheets and North Atlantic deep water circulation. Paleoceanography, 4:413-446.

Ruddiman, W. F., Cameron, D., and Clement, B. M., 1986. Sediment disturbance and correlation of offset holes drilled with the hydraulic piston corer: Leg 94. In Ruddiman, W. F., Kidd, R. B., Thomas, E., et al., Init. Repts. DSDP, 94: Washington (U.S. Govt. Printing Office), 615-634.

Ruddiman, W. F., Raymo, M. E., Martinson, D. G., Clement, B. M., and Backman, J., 1989. Pleistocene evolution: Northern Hemisphere ice sheets and North Atlantic Ocean. Paleoceanography, 4:353-412.

Saito, T., Burckle, L. H., and Hays, J. D., 1975. Late Miocene to Pleistocene biostratigraphy of equatorial Pacific sediments. In Saito, T., 
and Burckle, L. H. (Eds.), Late Neogene Epoch Boundaries: New York (Micropaleontology Press), 226-244.

Shaw, A. B., 1964. Time in Stratigraphy: New York (McGraw-Hill).

Shipboard Scientific Party, 1983. Sites 501 and 504: sediments and ocean crust in an area of high heat flow on the southern flank of the Costa Rica Rift. In Cann, J. R., Langseth, M. G., Honnorez, J., Von Herzen, R. P., White, S. M., et al., Init. Repts. DSDP, 69: Washington (U.S. Govt. Printing Office), 31-173.
Shipboard Scientific Party, 1988. Sites 677 and 678. In Becker, K., Sakai, H., et al., Proc. ODP, Init. Repts., 111: College Station, TX (Ocean Drilling Program), 253-346.

Date of initial receipt: 20 September 1988

Date of acceptance: 22 March 1989

Ms 111B-146

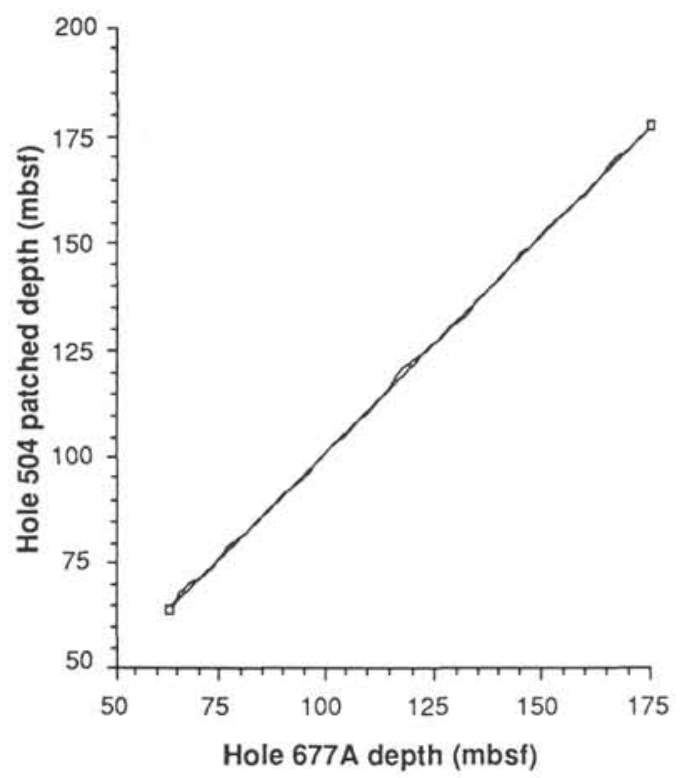

Figure 10. Mapping function produced by correlation of the calcium carbonate record from Hole 677A with the patched calcium carbonate record of Hole 504 using a full set of tie-lines and 39 coefficients. Coherence is 0.93 . Straight line indicates one-to-one correlation. 